Autonomous Robots 19, 193-214, 2005

\title{
Robust Control for Steer-by-Wire Vehicles
}

\author{
N. BAJÇINCA \\ Institute of Robotics and Mechatronics, DLR, Oberpfaffenhofen, Germany \\ naim.bajcinca@dlr.de \\ R. CORTESÃO \\ Institute of Systems and Robotics, ISR, Coimbra, Portugal \\ cortesao@isr.uc.pt \\ M. HAUSCHILD \\ Institute for Biomedical Engineering, University of Southern California, USA \\ markus.hauschild@usc.edu
}

\begin{abstract}
The design and analysis of steer-by-wire systems at the actuation and operational level is explored. At the actuation level, robust force feedback control using inverse disturbance observer structure and active observer algorithm is applied to enhance the robustness vs non-modelled dynamics and uncertain driver bio-impedance. At the operational level, the robustness aspects vs parameter uncertainties in vehicle dynamics and driver bioimpedance are issued and for a given target coupling dynamics between driver and vehicle the design task is converted to a model-matching problem. $H_{\infty}$ techniques and active observer algorithms are used to design the steer-by-wire controller. Robustness issues at both levels are covered by mapping stability bounds in the space of physical uncertain parameters.
\end{abstract}

Keywords: steer-by-wire, robust control, force control, inverse disturbance observer, active observer, modelmatching, robustness analysis

\section{Introduction}

Vehicle steering technology is evolving by substituting the mechanical and hydraulic subsystems with electrical equivalents to boost performance and enhance safety. The steer-by-wire technology provides essential advantages, such as simplified construction and higher design flexibility at the price of redundancy and safety measures. It introduces a complex steering technology consisting of computing units, sensors and actuators. Thereby the mechanical interface (steering column) between driver and vehicle is replaced by two electrical actuators which are coupled by a controller to provide the driver a desired steering feeling and the vehicle a desired steering response.
Apart from torque ripple and friction compensation, at the force feedback actuation level, the main challenge is to provide robustness with respect to uncertainties of the driver arms stiffness and inertia, and torque signals produced at the driver muscles, (Bajçinca et al., 2003a, 2003b). In order to cope with modelling errors, disturbances and parameter uncertainties, two novel control structures have been investigated: (a) inverse disturbance observer (IDOB), (Bajçinca and Bünte, 2005), a two degree-of-freedom structure which combines feedforward inversion and high-gain feedback, and (b) active observers (AOB), (Cortesão, 2003), a special modelreference adaptive control structure based on Kalman filtering. 
A steer-by-wire system is a typical master-slave system with neglectable time delay communication between the master and slave loop. Hence, it is natural to consider the transfer of the master-slave technology control to the steer-by-wire one. In this paper it will be shown that yet new design challenges emerge since: (a) the so-called intervening impedance between the driver (operator) and the vehicle (environment) has a relatively complex dynamics and it may include static nonlinearities (power assisted steer-bywire), and (b) the environment of steer-by-wire vehicles is non-passive, thus putting obstacles within the passivity design framework, which has been almost the traditional approach in master-slave system design.

Other contributions in this paper are: (a) modelmatching solution of the design problem using $H_{\infty}$ techniques and $\mathrm{AOB}$ formalism (Cortesão and Bajçinca, 2004) and (b) analysis of robust stability of a steer-by-wire system w.r.t main parametric uncertainties of the environment (driver arms stiffness, vehicle speed and tyre-road friction). It is shown that the lateral vehicle impedance is not robust passive for a non-zero vehicle speed. The output passivity excess of the controller may not compensate for the passivity shortage of the lateral vehicle dynamics. Thus, for a given target dynamics, passivity requirements may not be achievable. Yet the performance requirements may still be met while keeping robust stability. The robustness analysis methods in the presence of static sector non-linearities (boost-curve) are provided based on solving for the bounds of positive-realness of transfer matrix functions in parameter space. The latter approach may be useful for tuning of the power assistance boost-curve to guarantee robust absolute stability.

The paper is organized as follows. In Section 2 the basic coupling structure between the actuation and driver loop is investigated, a linear actuator model is presented, and robustness issues with respect to main uncertainties are tasked. Section 3 reveals the basic idea and features of the inverse disturbance control, shows its application on force feedback design, and finally provides a robustness analysis in parameter space of the operator bio-impedance. Section 4 gives a brief introduction of the active observer algorithms. Section 5 presents validation data for the force feedback controllers obtained by simulation and experimental tests. Section 6.1 steps in the operational (functional) level by discussing the target dynamics of steer-bywire systems. Section 6.2 discusses its environment, namely bio-mechanics of the driver arms and lateral vehicle dynamics. Section 6.3 describes the open-loop of a steer-by-wire system at the operational level. Section 6.4 presents the model-matching synthesis of a steer-by-wire controller using $H_{\infty}$ techniques and Section 6.5 using AOB formalism. Section 6.6 collects simulation results and finally, Section 7 provides the analysis of robust stability in the space of physical uncertain parameters.

\section{Steer-by-Wire Actuation}

\subsection{Actuation Modelling}

The interaction between the driver and a steer-bywire system can be realized in two different ways. In an admittance-like steer-by-wire control structure (the driver input is torque) the driver is provided force feedback in a direct way by a force-control loop. On the contrary, in an impedance-like controller structure (the driver input is steering-wheel angle) the interaction is realized by a position-control loop. While theoretically both of the schemes are equivalent, the direct force feedback scheme is more intuitive and is preferred in this article.

Figure 1 shows schematically the interaction of the driver and force feedback actuation loop. Note that the two loops are coupled by the signals $\tau_{f}$ and $\dot{\delta}_{l}$. The following listing clarifies the notation used in this scheme:

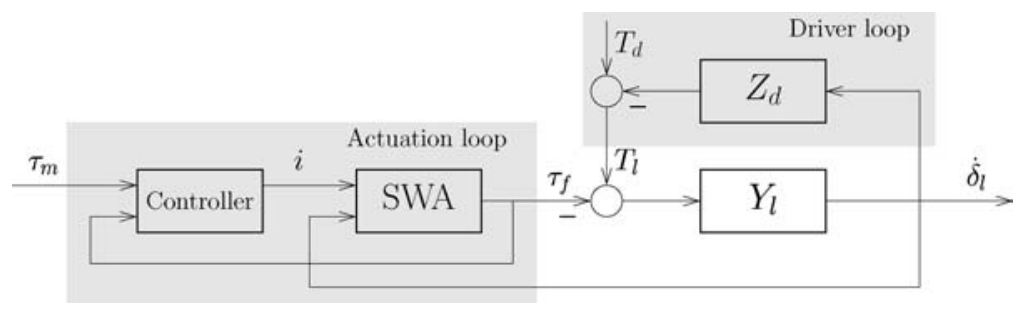

Figure 1. Coupling of actuation and driver loop. 
$T_{d}: \quad$ torque generated at the driver muscles

$T_{l}$ : net driver torque

$\delta_{l}$ : steering-wheel angle

$\tau_{m}:$ reference torque

$\tau_{f}:$ torque at the actuator output shaft

$Z_{d}$ : driving point impedance

$Y_{l}: \quad$ steering-wheel admittance $\left(=1 / J_{l} s\right)$

$i$ : motor current

If $\delta_{f}$ is the gear rotation angle, then the transferred torque over the gear is

$$
\tau_{f}=Z_{g}\left(\dot{\delta}_{f}-\dot{\delta}_{l}\right)
$$

where $Z_{g}=D_{g}+C_{g} / s$, and $D_{g}$ and $C_{g}$ stand respectively for the damping and stiffness of the gear. In other words, the motion of steering wheel induces a feedback torque component in the actuation loop. The trouble here is that this torque component is corrupted by large model and parameter uncertainties of the driver loop in addition to the injected torque $T_{d}$. Torque control in the presence of these uncertainties represents the main challenge in this section.

A simple model for $\tau_{f}$ lumps the net impact of the driver loop into a single exogenous disturbance by the following interpretation of (1)

$$
\tau_{f}=\left.\tau_{f}\right|_{\dot{\delta}_{l} \equiv 0}-Z_{g} \dot{\delta}_{l}=\tau_{f, \infty}+\text { disturbance }
$$

where $\tau_{f, \infty}=\left.\tau_{f}\right|_{\dot{\delta}_{l} \equiv 0}$ stands for the torque of the situation with the fixed steering wheel. This index denotation addresses the corresponding fictive physical situation with infinity load, that is operator arms impedance. The open-loop actuation can now be described by the model

$$
\tau_{f}=G_{\infty} i+G_{l} \dot{\delta}_{l}
$$

where $G_{\infty}$ represents the model of the fixed steeringwheel, and $G_{l}$ the open-loop transfer function from $\dot{\delta}_{l}$ to $\tau_{f}$. Note that $G_{l}$ differs from $Z_{g}$ due to the internal torque feedback within the harmonic-drive gear.

The advantage of this approach lies in the avoidance of the uncertainties of the driver loop into the plant model. It is also convenient that the impact of the driver loop is described by an additive output disturbance, that is by the term $G_{l} \dot{\delta}_{l}$, as denoted in (2).

An alternative interesting modelling approach is to try to discriminate the effect that result from the driver impedance $Z_{d}$ by a model of the form

$$
\left.\tau_{f}=\left.\tau_{f}\right|_{Z_{d}=0}+\text { (term due to } Z_{d}\right) .
$$

The first summand corresponds to the situation of the free (released) steering wheel. The reader may easily check that the condition $Z_{d}=0$ is physically equivalent to $T_{l}=0$. Hence (4) the can be written as

$$
\tau_{f}=G_{0} i+G_{t} T_{l}
$$

where $G_{0}$ corresponds to the open-loop model with zero operator impedance. Again $G_{t} T_{l}$ will be considered as an exogenous output disturbance.

The relationship between this and the model (3) can be directly computed from Fig. 1

$$
G_{0}=\frac{G_{\infty}}{1+Y_{l} G_{l}} \quad \text { and } \quad G_{t}=\frac{Y_{l} G_{l}}{1+Y_{l} G_{l}}
$$

The description (5) enjoys the same advantages as (3) with regard to uncertainties of the driver loop. However, the two models describe diametrally opposite situations: $G_{0}$ the zero load (released steering wheel), and $G_{\infty}$ the infinity load (fixed steering wheel).

\subsection{Driver Loop}

For the sake of simplicity set $\tau_{m}=0$ in Fig. 1. Then the impact of the actuation loop into the driver loop for the fixed steering wheel model (3) can be schematized as shown in Fig. 2(a). This structure is clear, since with $\tau_{m}=0$, the torque on the output shaft equals $\tau_{f}=$ $S_{\infty} G_{l} \dot{\delta}_{l}$, where $S_{\infty}$ represents the sensitivity function of the actuation closed loop with the description (3). In other words, the impact of the actuation loop into the driver loop can be weighted by its sensitivity function $S_{\infty}$.

Similarly for the free steering wheel model (5), the driver loop with $\tau_{m}=0$ transforms to Fig. 2(b). Here $S_{0}$ stands again for the sensitivity function from the disturbance $G_{t} T_{l}$ to $\tau_{f}$ in the closed loop.

The investigation of the driver loop is of interest particularly for robust stability analysis with respect to uncertainties in the operator bio-impedance $Z_{d}$. A simple robustness condition results from the observation that the feedback loop consisting of $Y_{l}$ and $Z_{d}$ is naturally robust stable, irrespectively on parameter uncertainties in $Z_{d}$, since both $Z_{d}$ and $Y_{l}$ are passive. In other words, for robust stability the condition $S_{\infty}=0$ 


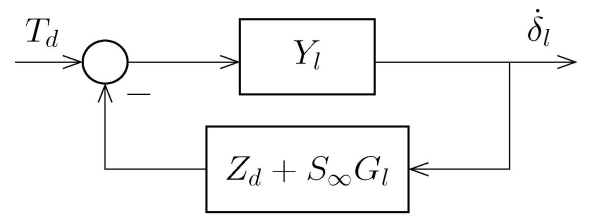

(a) Fixed steering wheel model description

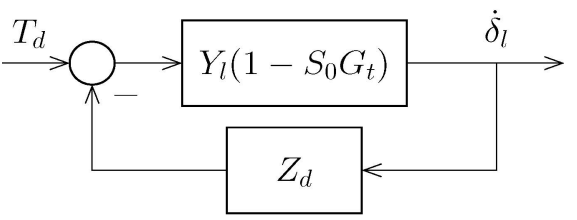

(b) Free steering wheel model description

Figure 2. Driver loop.

in Fig. 2(a) is sufficient. The same observation holds also for the structure Fig. 2(b) if $S_{0}=0$. Namely, for $S_{0}=S_{\infty}=0$ the two structures in Fig. 2 become identical. The operator feels then basically just the inertia of the steering wheel and the driver loop decouples from the actuation loop. However $S_{\infty}$ must increase at higher frequencies, and the term $Z_{d}+S_{\infty} G_{l}$ may run the loop in Fig. 2(a) unstable. It is intuitive that the situation is more critical for higher driver arms impedance e.g. if the operator holds the steering wheel stiffer, see Section 3.6.

Following the tradition in the teleoperation literature the simple model for the operator arms impedance

$$
Z_{d}=\frac{C_{d}}{s}+D_{d}+J_{d} s
$$

is here accepted, where $C_{d}, D_{d}$ and $J_{d}$ are respectively the stiffness, damping and inertia of the operator arms about the center of the steering wheel. We

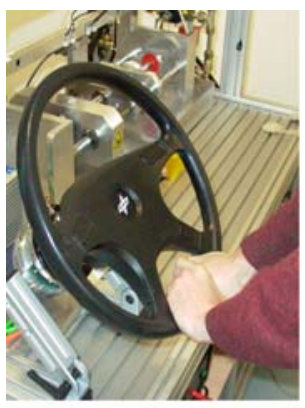

(a)

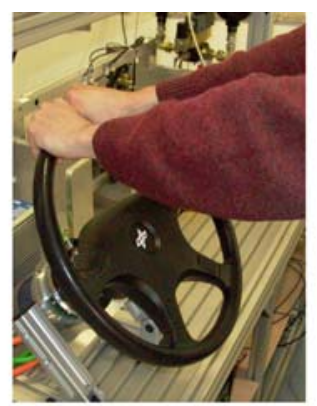

(b)

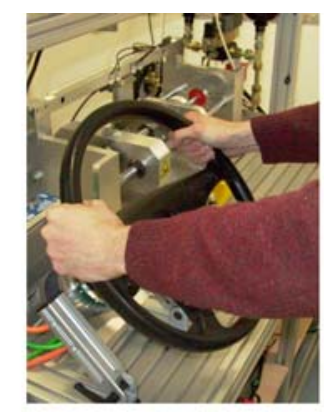

(c)

expect that the main uncertainties in (7) arise in the stiffness $C_{d}$ and moment of inertia $J_{d}$. Variations in $C_{d}$ are mainly due to different neuro-muscular operator arm systems, driving style and experience, operator age, driving situations etc. On the other hand, the moment of inertia $J_{d}$ is very sensitive on how the steering wheel is held. For instance, it has been experimentally tested that $J_{d}$ is the lowest if the steering wheel is grasped as shown in Fig. 3(a) and the largest for the situation in Fig. 3(d). The latter grasping turns out to be the most critical one also in terms of stiffness $C_{d}$. Namely, the stiffness induced by the operator is essentially higher compared to other situations in Fig. 3.

\subsection{Actuator Hardware}

The scheme of the steer-by-wire force feedback actuator is shown in Fig. 4. Its main components are a brushless DC motor, a harmonic-drive gear and a torque sensor. The harmonic-drive gear consists of flexspline, wave generator and circular spline. Note that the motor shaft is connected to the wave generator and the circular spline to the motor housing, which are both fixed at the console. The flexspline is constructed in the form of an ellipse so that in a given position it touches the circular spline only at two points. Its task is to transfer the motion introduced at the wave generator to the output shaft. Since the flexspline has two teeth less than the circular spline, one revolution of the input causes relative motion between the flexspline and the circular spline equal to two teeth. With the circular spline rotationally fixed, the flexspline rotates in the opposite direction to the input at a reduction ratio equal to one-half the number of teeth on the flexspline. Typical characteristics of a harmonic-drive

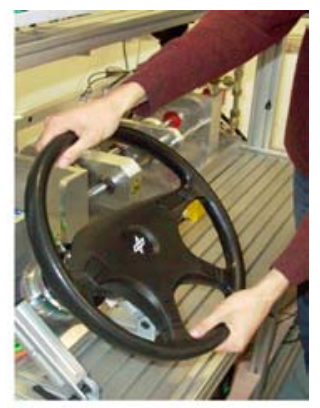

(d)

Figure 3. Uncertain moment of inertia depending on steering wheel grasping. 


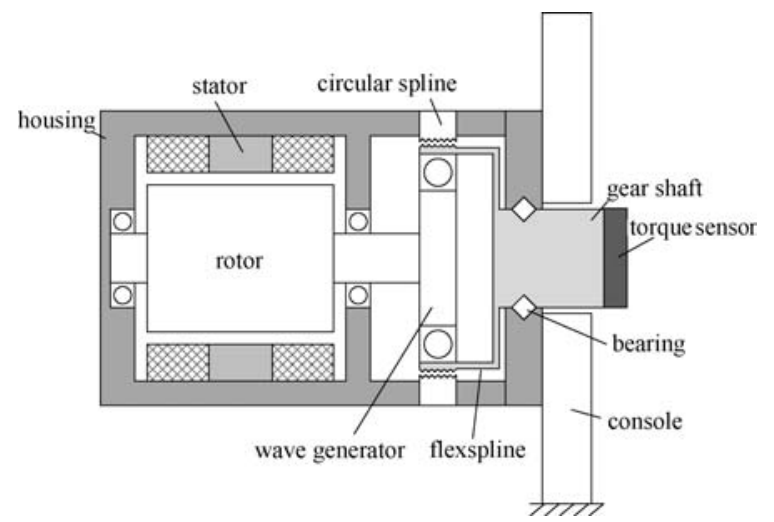

Figure 4. Force feedback actuator.

gear are high positioning accuracy, high gear ration and virtually no backlash, but with periodic torque ripples. The fundamental periodic frequency corresponds to twice the frequency of the motor shaft speed, resulting due to touching points between the flexspline and circular spline.

The torque sensor is based on strain gauges. This operating principle implies that an additional elasticity is introduced into the mechanical system which accounts for approximately one third of the entire gear elasticity. The use of two independent measurement bridges provides redundancy and also allows the compensation of temperature changes and other disturbances. As seen in Fig. 4, the sensor is mounted directly on the gear output shaft.

\subsection{Linear Actuator Model}

The conversion of motion over an ideal harmonic drive gear with fixed circular spline can be described by the following equations

$$
\begin{aligned}
\tau_{f} & =n \tau_{w} \\
n & =\delta_{w} / \delta_{f}
\end{aligned}
$$

where $n$ represents the transmission and the indices $\mathrm{f}$, and $w$ stand for flexspline and wave generator, respectively.

A simplified linear mass-stiffness-mass system shown in Fig. 5 can be used for design-purpose modelling of the force feedback actuator. Thereby the following three assumptions are set: (a) the gear moment of inertia is concentrated in front of the gear transmis-

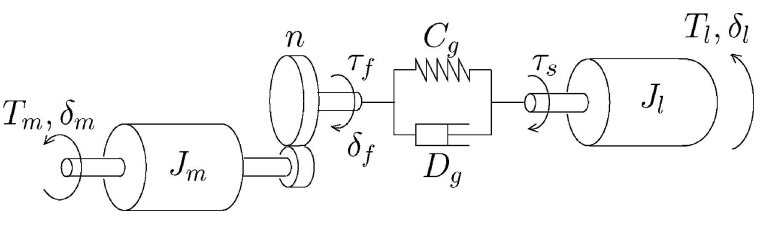

Figure 5. Mass-stiffness-mass model.

sion ratio $n$, and (b) a torsion stiffness $C_{g}$ with a low damping $D_{g}$ appear after the gear transmission ratio. According to Fig. 4 the sensor is connected in series with the flexspline, that is it is located at the signal $\tau_{s}$ in Fig. 5. However the sensor is omitted therein, since its stiffness is much higher than that of the gear.

The equations which describe the dynamics of the mass-stiffness-mass model in Fig. 5 are then easily derived

$$
\begin{aligned}
J_{m} \ddot{\delta}_{m} & =T_{m}-\frac{\tau_{s}}{n} \\
\tau_{s} & =-J_{l} \ddot{\delta}_{l}+T_{l} \\
\tau_{s} & =C_{g}\left(\delta_{l}+\frac{\delta_{m}}{n}\right)+D_{g}\left(\dot{\delta}_{l}+\frac{\dot{\delta}_{m}}{n}\right) .
\end{aligned}
$$

The transmission equations of harmonic drive (8) and (9) are included here by $\tau_{s}=\tau_{f}$ and $\delta_{m}=\delta_{w}$. Substituting $T_{m}=K_{m} i$, where $K_{m}$ stands for the motor torque constant, and $i$ for the motor current, the latter equations read

$$
\tau_{f}=\frac{n\left(C_{g}+D_{g} s\right)\left(K_{m} J_{l} i+n J_{m} T_{l}\right)}{n^{2} J_{m} J_{l} s^{2}+\left(J_{l} D_{g}+n^{2} J_{m} D_{g}\right) s+n^{2} J_{m} C_{g}+J_{l} C_{g}} .
$$

To compute $\tilde{G}_{\infty}$ in (3) set $T_{l}=0$ and let $J_{l} \rightarrow \infty$ in (13)

$$
\tilde{G}_{\infty}=\frac{b_{1} s+b_{0}}{a_{2} s^{2}+a_{1} s+a_{0}}
$$

with

$$
\begin{aligned}
& b_{0}=n K_{m} C_{g} \\
& b_{1}=n K_{m} D_{g} \\
& a_{0}=C_{g} \\
& a_{1}=D_{g} \\
& a_{2}=n^{2} J_{m} .
\end{aligned}
$$


To compute $G_{l}$ in (3) set $T_{m}=0$ in (10) and substitute it in (12)

$$
G_{l}=\frac{c_{2} s^{2}+c_{1} s}{a_{2} s^{2}+a_{1} s+a_{0}}
$$

with

$$
\begin{aligned}
& c_{1}=n^{2} J_{m} C_{g} \\
& c_{2}=n^{2} J_{m} D_{g} .
\end{aligned}
$$

The two transfer functions $G_{0}$ and $G_{t}$ in the model (5) are directly computed from (13)

$$
\tilde{G}_{0}=\frac{b_{1}^{\prime} s+b_{0}^{\prime}}{a_{2}^{\prime} s^{2}+a_{1}^{\prime} s+a_{0}^{\prime}}
$$

with

$$
\begin{aligned}
& b_{0}^{\prime}=n K_{m} J_{l} C_{g} \\
& b_{1}^{\prime}=n K_{m} J_{l} D_{g} \\
& a_{0}^{\prime}=\left(J_{l}+n^{2} J_{m}\right) C_{g} \\
& a_{1}^{\prime}=\left(J_{l}+n^{2} J_{m}\right) D_{g} \\
& a_{2}^{\prime}=n^{2} J_{m} J_{l}
\end{aligned}
$$

and

$$
G_{t}=\frac{c_{1}^{\prime} s+c_{0}^{\prime}}{a_{2}^{\prime} s^{2}+a_{1}^{\prime} s+a_{0}^{\prime}}
$$

with

$$
\begin{aligned}
& c_{0}^{\prime}=n^{2} J_{m} C_{g} \\
& c_{1}^{\prime}=n^{2} J_{m} D_{g} .
\end{aligned}
$$

\section{Inverse Disturbance Observer}

\subsection{Basic Idea}

The inverse disturbance observer (IDOB) structure, (Bajçinca and Bünte, 2005), in its basic form is shown in Fig. 6. Identify here the plant $G$, and the two design parameters (two degrees of freedom): $\tilde{G}^{-1}$ the approximate inverse of $G$, and the $Q$-filter. The transfer functions from the reference $r$ to the plant input $u\left(G_{r u}\right)$ and its output $y\left(G_{r y}\right)$ are easily obtained from Fig. 6

$$
G_{r u}=\frac{\tilde{G}^{-1}}{1-Q\left(1-G \tilde{G}^{-1}\right)}
$$

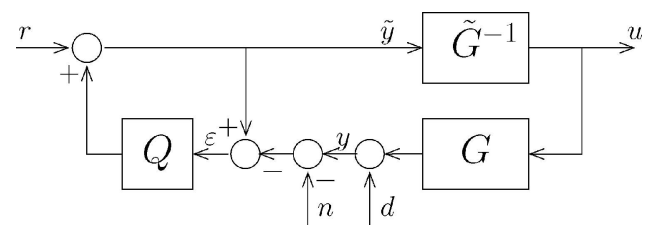

Figure 6. IDOB control structure.

$$
G_{r y}=\frac{G \tilde{G}^{-1}}{1-Q\left(1-G \tilde{G}^{-1}\right)} .
$$

To present the basic idea of the IDOB control structure introduce the notation:
(A): $Q=1$
(infinity-gain feedback)
$(B): \quad \tilde{G}^{-1}=G^{-1}$
(feedforward exact inversion)
$(C): G_{r y}=1$
(perfect tracking).

Then (18) and (19) read directly

$(A) \vee(B) \Rightarrow(C)$.

In practice, none of the conditions $(A)$ and $(B)$ is realizable for all frequencies. In fact, $(A)$ would destabilize any practical loop, so $Q$ must ultimately roll-off for high-frequencies. Further, the feedforward exact inversion as defined in $(B)$ fails at least for high-frequencies. Hence, the conditions $(A)$ and $(B)$ make sense only in the operational frequency bandwidth. Even then the two conditions must be weakened due to implementation limitations to
$\left(A^{\prime}\right): \quad Q \approx 1$
(high-gain feedback)
$\left(B^{\prime}\right): \quad \tilde{G}^{-1} \approx G^{-1}$
(feedforward inversion).

However it is important that though weakened the conditions $\left(A^{\prime}\right)$ and $\left(B^{\prime}\right)$ collaborate, that is, they contribute independently towards situation $(C)$. For $Q \approx 1$ due to the positive feedback in the $Q$-loop in Fig. 6 a high-gain controller results. If $\tilde{G}^{-1} \approx G^{-1}$, then exact feedforward inversion is approximately realized so the $Q$-feedback loop is almost idle. Therefore, IDOB control structurally unifies the high-gain feedback and the feedforward exact inversion principle, (20).

\subsection{Robustness}

Essential implications for the IDOB structure are provided by the sensitivity $S=y / d$ and complementary 
sensitivity $T=y / n$ functions

$$
\begin{aligned}
S & =\frac{1-Q}{1-Q\left(1-G \tilde{G}^{-1}\right)} \\
T & =\frac{Q G \tilde{G}^{-1}}{1-Q\left(1-G \tilde{G}^{-1}\right)} .
\end{aligned}
$$

The two are constrained by the fundamental algebraic condition $S+T=1$. Also $S=1 /(1+L)$ and $T=L /(1+L)$, where $L$ stands for the loop transfer function

$$
L=\frac{Q G \tilde{G}^{-1}}{1-Q} .
$$

It is convenient to define also the three latter functions for the situation that corresponds to feedforward perfect inversion (condition $(B)$ )

$$
\tilde{S}=1-Q, \quad \tilde{T}=Q, \quad \text { and } \quad \tilde{L}=\frac{Q}{1-Q} .
$$

One important implication of sensitivity function is related to the error $e=r-y$ dynamics, refer to Eq. (27). For typical applications requiring zero DC error tracking, $S(0)=0$ must hold, implying $Q(0)=1$. The latter condition produces always a pole at $s=0$ in the expression $1 /(1-Q)$. Hence according to (23) the pole $s=0$ appears also in the loop transfer function $L$.

In practice, $\tilde{G}^{-1}$ can never provide exact inversion of the plant $G$. Therefore it is natural to introduce the notion of imperfect inversion. For the sake of simplicity consider the multiplicative imperfection

$$
G=\tilde{G}\left(1+W_{2} \Delta\right)
$$

where $W_{2}$ is a proper stable weighting function and $\|\Delta\|_{\infty}<1$ represents a stable unstructured disk-like uncertainty. Typically, $W_{2}$ increases with frequency due to model mismatching.

The problem explored here is: assuming that IDOB closed loop with perfect inversion (condition $(\mathrm{B})$ ) is stable, how big is the minimal imperfection that destabilizes the IDOB loop? In Bajçinca and Bünte (2005) it is shown that robust stability is guaranteed if and only if the condition

$$
\left\|W_{2} Q\right\|_{\infty}<1
$$

holds. Equation (26) sets stability constraints in the interaction between the imperfection $W_{2}$ and the design parameter $Q$. It has a simple and elegant geometrical interpretation in terms of the Bode plots of $Q$ and $W_{2}$. Namely, the IDOB structure with the imperfection $W_{2}$ is internally stable iff the magnitude Bode plot of the function $W_{2}^{-1}$ lies above that of $Q$. Recall that $\left|W_{2}\right|^{-1}$ rolls off toward zero at high frequencies, thus putting a limitation for the bandwidth of the filter $Q$.

\subsection{Tracking}

Asymptotic tracking is defined as the ability of the control loop to drive $y \rightarrow r$, that is, $e \rightarrow 0$ as $t \rightarrow \infty$. For instance, if $r$ is a step, then due to

$$
\frac{e}{r}=S\left(1-G \tilde{G}^{-1}\right)
$$

the function $S$ must have a zero at $s=0$, which is equivalent to $Q(0)=1$. For example this is fulfilled by $Q=1 /(\tau s+1)$. Now consider a set of sinusoidal inputs confined within some frequency bandwidth. Let $W_{p}$ weight a desired tracking response in (27) in the sense that $\|e\|_{2}<\left\|W_{p}\right\|_{\infty}^{-1}\|r\|_{2}$. For larger $\left|W_{p}\right|$ the tracking performance improves. However, it is intuitive that for sufficiently large imperfections $W_{2} \Delta$, the performance set by $W_{p}$ may get lost. It is thus important to set up the conditions for meeting the performance set by $W_{p}$ in the presence of the imperfections $W_{2}$. Therefore, substitute (25) into (27) to get $\left|S W_{1} \Delta\right|<1, \forall \omega$, or equivalently

$$
\left\|W_{1} S\right\|_{\infty}<1
$$

where we switch to the notation $W_{1}=W_{p} W_{2}$. This equation can be further manipulated, see (Bajçinca and Bünte, 2005), provided that internal stability condition $\left\|W_{2} Q\right\|<1$ holds to

$$
\left\|\left|W_{1}(1-Q)\right|+\left|W_{2} Q\right|\right\|_{\infty}<1
$$

Equation (29) is an elegant description of simplicity and efficiency of the IDOB control structure. It represents the basic equation for the loopshaping design by $Q$. It can be shown that thereby a very simple $Q$ results (loopshaping details are avoided here). Namely, for operational frequencies, where $\left|W_{1}\right| \gg 1>\left|W_{2}\right|$ holds, the condition $Q \approx 1$ results, and for high frequencies $\left(\left|W_{2}\right| \gg 1>\left|W_{1}\right|\right), Q \approx 0$ results. In general, it is easy to define such a $Q$, e.g. $Q=1 /(\tau s+1)$. The only design parameter here is basically its bandwidth. For its design (29) should be used. Note that, 
for better performance the term $\left|W_{1}(1-Q)\right|$ in (29) requires a high bandwidth, which is however compelled by the stability term $\left|W_{2} Q\right|$. Thus, the designer should meet a compromise between these two conflicting specifications.

\subsection{Fixed Steering Wheel Inversion}

The inverse disturbance observer control structure for the force feedback control is shown in Fig. 7. The transfer function $\tilde{G}_{\infty}^{-1}$ represents here the inverse of the linear model of the fixed actuator. Identify in the grayed block the plant model defined in (3). Since the gear does not rotate the bearing friction component is zero, thus resulting in a good matching between $\tilde{G}_{\infty}$ and $G_{\infty}$. Hence the feedforward inversion by $\tilde{G}_{\infty}^{-1}$ is almost exact, which in turn strongly supports IDOB control due to the good robustness of the latter with respect to output disturbances. Namely, it has been already noted that the control strategy here is based on lumping of uncertainties in the fictive output disturbance $G_{l} \dot{\delta}_{l}$.

The discussion in Section 2.4 serves to design $\tilde{G}_{\infty}^{-1}$. For the design of $Q$ the robust stability of the loop in Fig. 2(a) with respect to the uncertainties in $Z_{d}$ is to be considered, see Section 3.6. Due to the relative degree one of $\tilde{G}_{\infty}^{-1}$, a first order $Q$ filter is used

$$
Q=\frac{1}{\tau s+1}
$$

\subsection{Free Steering Wheel Inversion}

The control structure based on the inversion of the free steering wheel setup described by (5) is shown in Fig. 8. The transfer functions $\tilde{G}_{0}$ and $G_{t}$ are computed in Section 2.4. The $Q$ filter is given the form (30). Again its bandwidth is determined by computing the

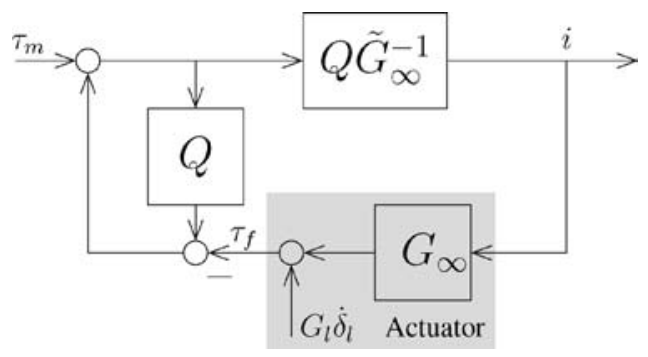

Figure 7. Force feedback control with fixed steering wheel inversion.

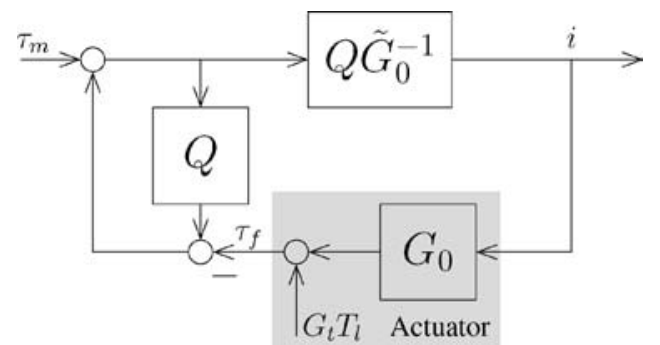

Figure 8. Force feedback control with free steering wheel inversion.

stability charts in the parameter space of $C_{d}, J_{d}$ and $\tau$, see below.

\subsection{Robustness Analysis}

The basic task here is to design the parameter $\tau$ (that is, the controller bandwidth $1 / \tau$ ) such that the force feedback actuation system remains stable under all parameter uncertainties in the driver arms stiffness $C_{d}$ and inertia $J_{d}$. Therefore consider the loops in Fig. 2. The characteristic equation of the loop Fig. 2(a) reads

$$
1+Y_{l}\left(Z_{d}+S_{\infty} G_{l}\right)=0
$$

and for that in Fig. 2(b)

$$
1+Y_{l} Z_{d}\left(1-S_{0} G_{t}\right)=0
$$

Assuming that perfect inversion holds in the structures in Figs. 7 and 8 according to (24)

$$
S_{\infty}=\tilde{S}_{\infty}=1-Q, \quad S_{0}=\tilde{S}_{0}=1-Q
$$

Substitution of the latter equation in (31) and (32) results in an equation with three uncertain parameters $J_{d}, C_{d}$ and $\tau$. The resulting Hurwitz stability bounds in the space of parameters $C_{d}, J_{d}$ and $\tau$ are shown in Fig. 9. Observe in Fig. 9(b) and (e) that for a given inertia $J_{d}$ a large enough stiffness $C_{d}$ turns the system unstable. This effect has been clearly observed in all experimental tests. 


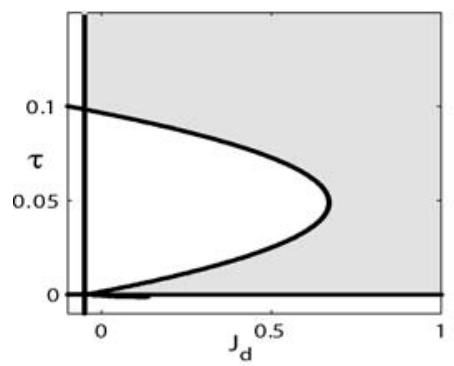

(a) $C_{d}=100$

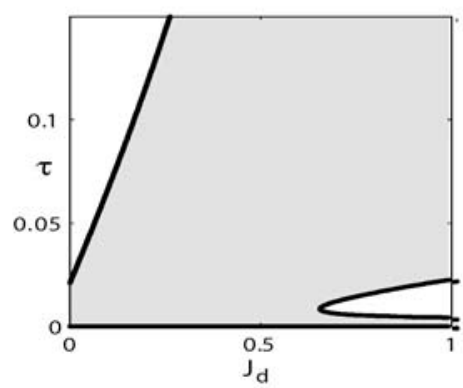

(d) $C_{d}=100$

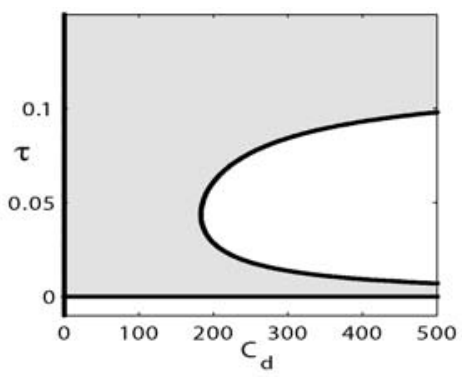

(b) $J_{d}=0.1$

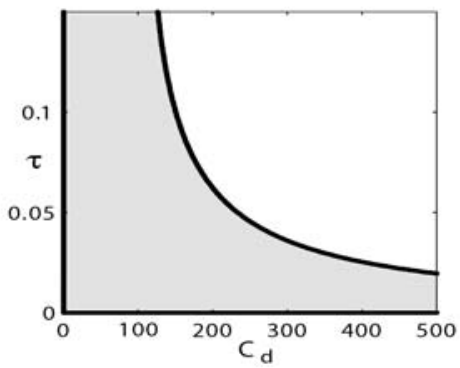

(e) $J_{d}=0.1$

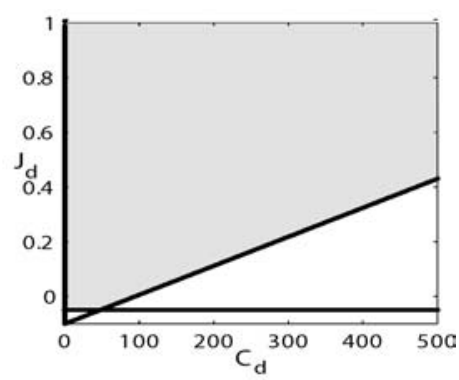

(c) $\tau=0.01$

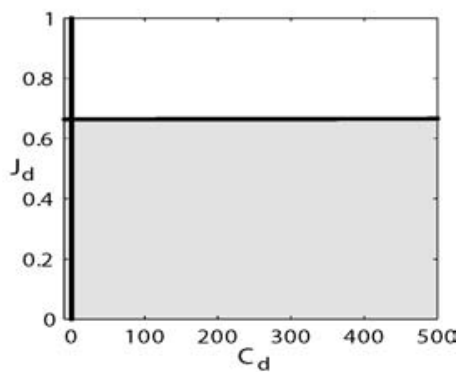

(f) $\tau=0.01$

Figure 9. Stability bounds in the parameter space of $C_{d}, J_{d}$ and $\tau$ for IDOB control based on fixed (first row) and free (second row) steering wheel inversion with respective controllers in Figs. 7 and 8. All variables are in SI units. The stable region is in gray.

\section{AOB Based Control}

A linear system represented in state space by

$$
\left\{\begin{array}{l}
x_{r, k}=\Phi_{r} x_{r, k-1}+\Gamma_{r} u_{k-1} \\
y_{k}=C_{r} x_{r, k}
\end{array}\right.
$$

can be controlled through state feedback (e.g. optimal control, adaptive control, deadbeat control and "pure" pole placement control). In practice, the main problem of this approach is that (34) does not represent exactly the real system. In fact, unmodeled terms including noise, higher order dynamics, parameter mismatches, couplings and unknown disturbances are not addressed in the control design. Therefore, it is necessary to develop a control structure that can deal with them, so that the overall system may have the desired behavior. The AOB state space control design satisfies these requirements. The main goal of the $\mathrm{AOB}$ is to fit a physical system (i.e. its input/output behavior) into a linear mathematical model, rather than to fit a mathematical model into a physical system. To accomplish this goal, a description of the system (closed loop and open loop) is necessary. A special Kalman Filter (KF) has to be designed. The motivation for this special KF is based on:

(1) A desired closed loop system for the state estimation.

(2) An extra equation to estimate an equivalent disturbance referred to the system input. An active state $p_{k}$ (extra-state) is introduced to compensate unmodeled terms, providing a feedforward compensation action.

(3) The stochastic design of the Kalman matrices $Q$ and $R$ for the AOB context. Model reference adaptive control appears if $Q_{x_{r, k}}$ is much smaller than $Q_{p_{k}}$. In this case, the estimation for the system state follows the reference model. Everything that does not fit in the $x_{r, k}$ model goes to $p_{k}$.

In the sequel, the first-order $\mathrm{AOB}$ algorithm ${ }^{1}$ will be described. Controlling the system of (34) through state feedback from an observer and inserting $p_{k}$ in the loop, the overall system can be described by

$\left[\begin{array}{c}x_{r, k} \\ p_{k}\end{array}\right]=\left[\begin{array}{cc}\Phi_{r} & \Gamma_{r} \\ 0 & 1\end{array}\right]\left[\begin{array}{c}x_{r, k-1} \\ p_{k-1}\end{array}\right]+\left[\begin{array}{c}\Gamma_{r} \\ 0\end{array}\right] u_{k-1}+d_{k}$ 
and

$$
y_{k}=C_{a}\left[\begin{array}{ll}
x_{r, k-1} & p_{k-1}
\end{array}\right]^{T}+n_{k}
$$

where

$$
u_{k-1}=r_{k-1}-\left[\begin{array}{ll}
L_{r} & 1
\end{array}\right]\left[\begin{array}{c}
\hat{x}_{r, k-1} \\
\hat{p}_{k-1}
\end{array}\right] .
$$

The stochastic inputs $d_{k}$ and $n_{k}$ represent respectively model and measure uncertainties. The state estimate of (35) is based on the desired closed loop (i.e. $\hat{p}_{k}=p_{k}$ and $\hat{x}_{r, k}=x_{r, k}$ ). It is

$$
\begin{aligned}
{\left[\begin{array}{c}
\hat{x}_{r, k} \\
\hat{p}_{k}
\end{array}\right]=} & {\left[\begin{array}{cc}
\Phi_{r}-\Gamma_{r} L_{r} & 0 \\
0 & 1
\end{array}\right]\left[\begin{array}{c}
\hat{x}_{r, k-1} \\
\hat{p}_{k-1}
\end{array}\right] } \\
& +\left[\begin{array}{c}
\Gamma_{r} \\
0
\end{array}\right] r_{k-1}+K_{k}\left(y_{k}-\hat{y}_{k}\right)
\end{aligned}
$$

with

$$
\hat{y}_{k}=C_{a}\left(\left[\begin{array}{cc}
\Phi_{r}-\Gamma_{r} L_{r} & 0 \\
0 & 1
\end{array}\right]\left[\begin{array}{c}
\hat{x}_{r, k-1} \\
\hat{p}_{k-1}
\end{array}\right]+\left[\begin{array}{c}
\Gamma_{r} \\
0
\end{array}\right] r_{k-1}\right)
$$

and

$$
C_{a}=\left[\begin{array}{ll}
C_{r} & 0
\end{array}\right]
$$

The Kalman gain $K_{k}$ reflects the uncertainty associated to each state based on model and measure uncertainties. It is computed from

$$
K_{k}=P_{1 k} C_{a}^{T}\left[C_{a} P_{1 k} C_{a}^{T}+R_{k}\right]^{-1}
$$

with

$$
P_{1 k}=\Phi_{n} P_{k-1} \Phi_{n}^{T}+Q_{k}
$$

and

$$
P_{k}=P_{1 k}-K_{k} C_{a} P_{1 k}
$$

$\Phi_{n}$ is the augmented open loop matrix

$$
\Phi_{n}=\left[\begin{array}{cc}
\Phi_{r} & \Gamma_{r} \\
0 & 1
\end{array}\right]
$$

$Q_{k}$ is the system noise matrix and represents model uncertainty. It is given by

$$
Q_{k}=\left[\begin{array}{cc}
Q_{x_{r, k}} & 0 \\
0 & Q_{p_{k}}
\end{array}\right] .
$$

The measurement noise matrix $R_{k}$ represents measure uncertainty. $P_{k}$ is the mean square error matrix. Its initial value should reflect at least the uncertainty in the state estimation. It should not be lower than the initial matrix $Q_{k}$.

\section{Control Validation}

Validation data obtained by simulation and experimental tests are collected in this section for both control structures, IDOB and AOB. The respective plots are shown in Figs. 10 and 11. Thereby three test scenarios are examined for the IDOB controller: (a) robust tracking of a step-like and (c) sinusoidal reference, and (b) disturbance rejection of the operator torque on the steering wheel. For the driver torque rejection the reference torque is set to zero, and a step-like torque is applied on the steering wheel. The data are obtained for nominal, large, stiff, soft and zero operator arms impedance.

For the AOB control two scenarios have been investigated: (a) output disturbance rejection and (b) input tracking. Note that additionally the active state responses are shown. The experiments are done with nominal and infinity operator arms impedance.

\section{Design of Steer-by-Wire Systems}

\subsection{Target Dynamics}

The first step when designing the steer-by-wire steering dynamics is to set its reference, i.e. to give some answer to the natural question how a steer-by-wire system should feel like and how the vehicle should react on the driver steering command. In the sequel, such a desired system dynamics will be referred to as reference or target steering dynamics. Some straightforward target steering systems are, of course, the contemporary steering systems, s.a. Electrical (EPAS) or/and Hydraulic Power Assisted Steering (HPAS), Fig. 12. 


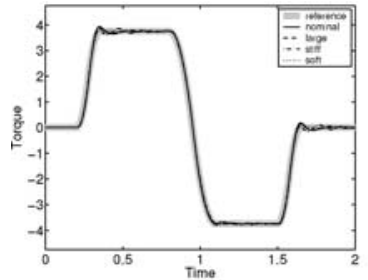

(a) step-like tracking

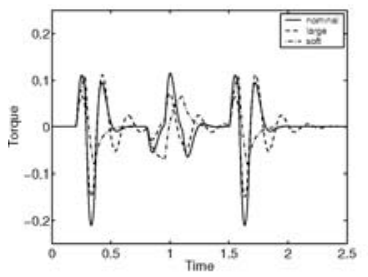

(e) driver torque rejection

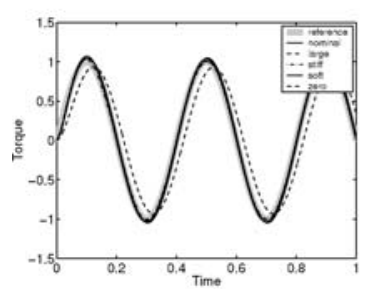

(i) sinus tracking

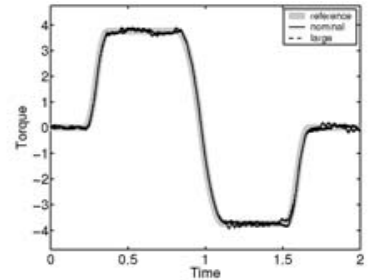

(b) step-like tracking

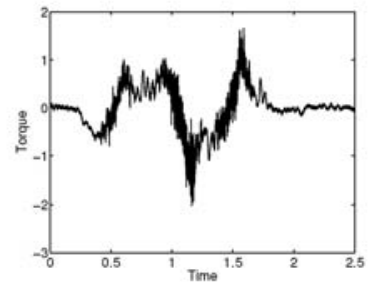

(f) driver torque rejection

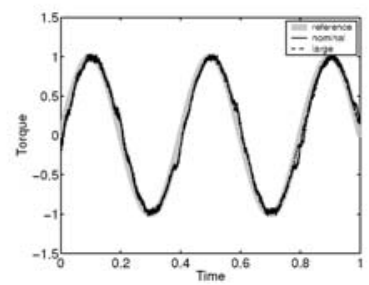

(j) sinus tracking

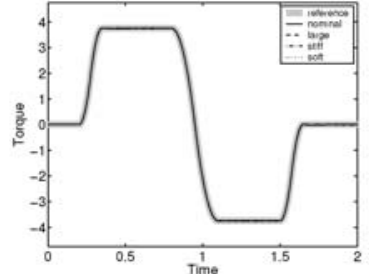

(c) step-like tracking

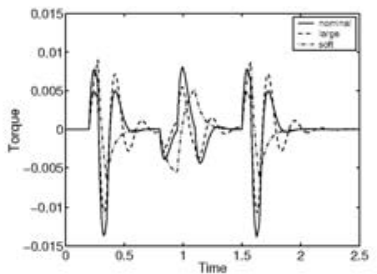

(g) driver torque rejection

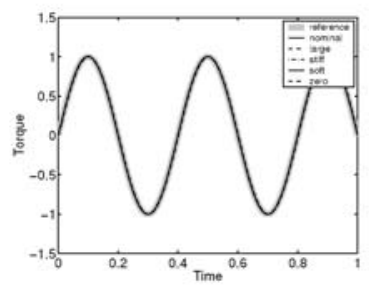

(k) sinus tracking

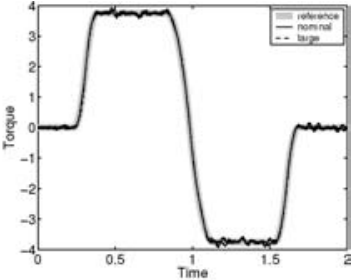

(d) step-like tracking

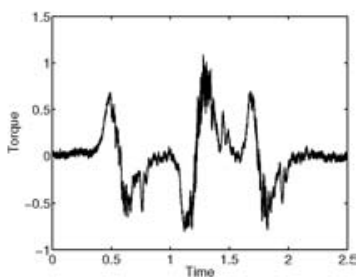

(h) driver torque rejection

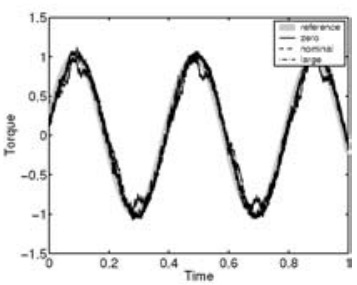

(1) sinus tracking

Figure 10. Simulation and experimental data with the IDOB controller. Column 1: Simulation data with structure in Fig. 7; Column 2: Experimental data with structure in Fig. 7; Column 3: Simulation data with structure in Fig. 8; Column 4: Experimental data with structure in Fig. 8. The variables are in SI units.
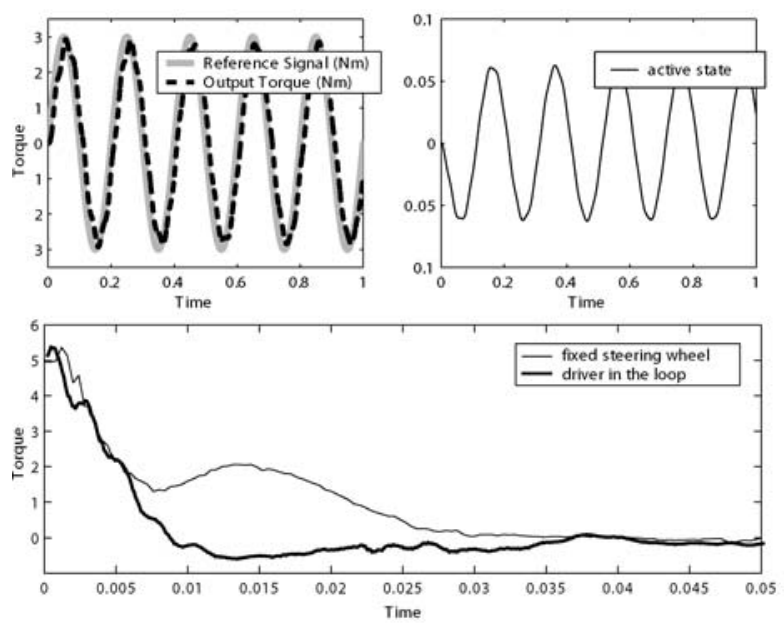

(a) Disturbance rejection
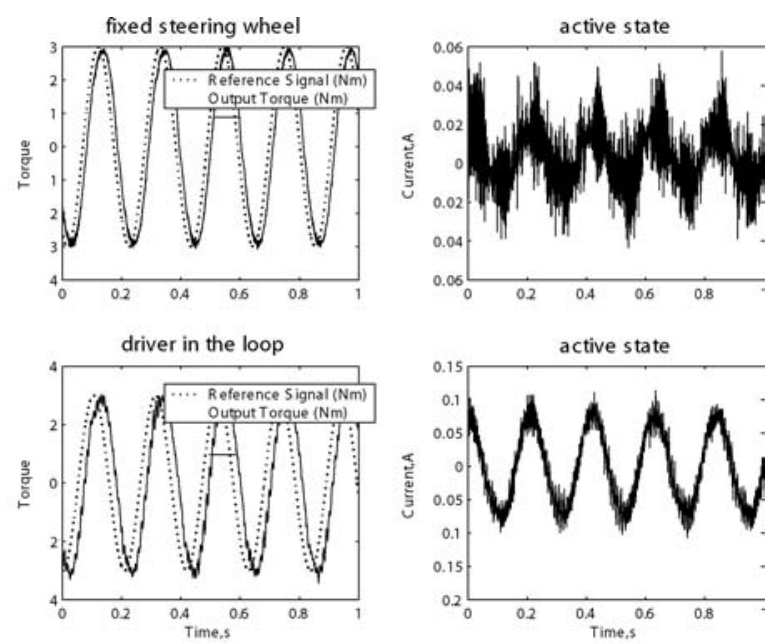

(b) Torque tracking

Figure 11. Simulation and experimental data with the AOB controller. The variables are in SI units. 


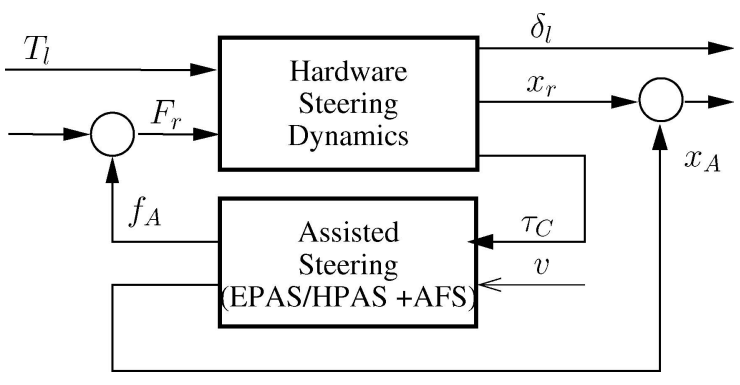

Figure 12. Steering system dynamics.

The relevant signals in Fig. 12 are

$\begin{array}{ll}T_{l}: & \text { torque on steering wheel } \\ F_{r}: & \text { steering rack force } \\ x_{r}: & \text { steering rack position } \\ \delta_{l}: & \text { steering wheel angle. }\end{array}$

Notice that steering systems comprehend assisting algorithms for independent force and position assistance. Usually the assisted torque steering filter is a nonlinear (parabolic) function of vehicle speed $v$ and steering column torsional angle, $\delta_{C}$ (the so-called boost curve). While torque assistance is mainly to increase the steering comfort, the steering angle (i.e. rack position) assistance is introduced for improvement of lateral and/or vertical vehicle dynamics.

The hardware steering dynamics in Fig. 12 includes the mechanical dynamics of the steering wheel, steering column, steering rack, power steering actuator, and that of the torque sensor on the steering column. Under assumption that it can be approximated by a linear system, i.e. the nonlinear gearing friction is neglected, its dynamics can be represented by the following twoinput two-output admittance system,

$$
\left[\begin{array}{l}
\dot{\delta}_{l} \\
\dot{x}_{r}
\end{array}\right]=\left[\begin{array}{ll}
y_{11} & y_{12} \\
y_{21} & y_{22}
\end{array}\right]\left[\begin{array}{l}
T_{l} \\
F_{r}
\end{array}\right]
$$

or by an equivalent hybrid or impedance representation. Since the steering column dynamics is included in the linear part of the steering dynamics, it is clear that in order to match the steering dynamics of a given target steering dynamics (e.g. EPAS) with a steer-by-wire technology, exactly this dynamics has to be reproduced by a suitable steer-by-wire actuation. The code which provides the nonlinear power assisted steering can be reused as it is, thus reducing the design problem of a steer-by-wire system into a linear control problem.

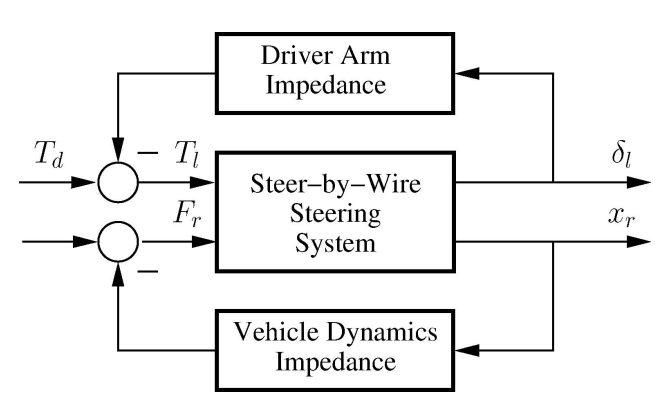

Figure 13. Interaction of a steer-by-wire system with its environment.

\subsection{System Uncertainties}

A steer-by-wire system can be classified as a masterslave robotic system, whereby the driver corresponds to the operator and the vehicle dynamics to its environment. Besides transparency (target dynamics matching) a basic design requirement of a steer-by-wire system is stability robustness particularly w.r.t. parametric uncertainties of driver arms stiffness ${ }^{2}\left(C_{d}\right)$, vehicle speed $(v)$ and road-tyre friction coefficient $(\mu)$. Though (see Fig. 13) the environment provides a negative feedback to a steer-by-wire system, it is important to investigate the robustness of such an interaction. In the next two sections the driver arm and the vehicle dynamics impedance are considered.

Bio-Mechanical Human Arm Impedance. To get an idea about the modelling of the biomechanics of the operator arms here are briefly collected some basic results of single-joint muscle system, which are usually used in teleoperation robotics. The main aim is to bring some physical understanding for the model (7) and discuss the major uncertainties therein.

The muscle actuator of the driver arm may be roughly modelled by a linear equation as follows

$$
\Delta x / \Delta t=g_{m \alpha} \Delta f_{\alpha}+g_{m f} f_{m}
$$

whereby

$\Delta x:$ internal muscle length contraction

$\Delta f_{\alpha}: \quad$ nerve excitation of the $\alpha$-neuron

$f_{m}$ : force acting on the muscle

$g_{m \alpha}:$ feedforward admittance

$g_{m f}:$ driving point admittance. 
Note that (47) contains a feedforward path due to $\alpha$-neuron excitation and a so-called driving point impedance for description of the muscle contraction in response to the load force $f_{m}$. The feedforward path of the muscular actuator driven by $\alpha$-efference represents an active system and is responsible for the adaption of the net arm impedance and control of arm position based on feedback of neural activation. This term corresponds to the signal $T_{d}$ in Fig. 1. The human arm impedance adaption process is associated with time delays ranging from 30 to $70 \mathrm{~ms}$ signal transmission delays thus resulting with a low bandwidth feedback loop 0.5-1.7 Hz, (Hogan, 1990).

Both terms $g_{m \alpha}$ and $g_{m f}$ depend on muscle stiffness, muscle damping and the net mass of the limb and interface element (e.g. joystick or steering wheel). For the purposes of this chapter especially important is the driving point admittance $g_{m f}$. A common modelling approach for $g_{m f}$ in the teleoperation robotics literature is a linear mass/damping/stiffness model of the form

$$
g_{m f}=\frac{1}{m_{o} s+d_{o}+c_{o} / s} .
$$

Measurement suggest, (Hogan, 1989), minimal incremental elbow stiffness of $2 \mathrm{Nm} / \mathrm{rad}$ and maximal of $400 \mathrm{Nm} / \mathrm{rad}$. On the other hand, the damping was estimated to be around $5.5 \mathrm{Ns} / \mathrm{m}$.

Lateral Vehicle Impedance. The car model which has been used for the investigations in this paper is the classical linearized single track model as illustrated in Fig. 14.

Its basic variables and geometric parameters are ${ }^{3}$

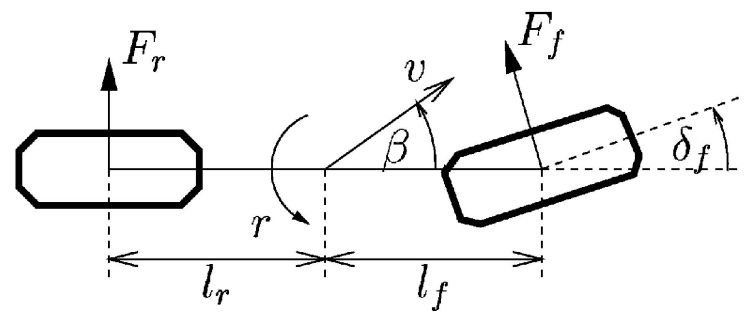

Figure 14. Single-track model.
$F_{f}\left(F_{r}\right)$ : lateral wheel force at front (rear) wheel

$r: \quad$ yaw rate

$\beta: \quad$ chassis side slip angle at

center of gravity $(\mathrm{CG})$

$v$ : magnitude of velocity vector at $\mathrm{CG}$ $(v>0, \dot{v}=0)$

$\ell_{f}\left(\ell_{r}\right): \quad$ distance from front (rear) axle to CG

$\delta_{f}$ : $\quad$ front wheel steering angle

The mass of the vehicle is $m$ and $J$ is the moment of inertia with respect to a vertical axis through the CG. For small steering angle $\delta_{f}$ and small side slip angle $\beta$, the linearized equations of motion are

$$
\begin{aligned}
m v(\dot{\beta}+r) & =F_{f}+F_{r} \\
m \ell_{f} \ell_{r} \dot{r} & =F_{f} \ell_{f}-F_{r} \ell_{r}
\end{aligned}
$$

The tire force characteristics are linearized as

$$
F_{f}\left(\alpha_{f}\right)=\mu c_{f 0} \alpha_{f}, \quad F_{r}\left(\alpha_{r}\right)=\mu c_{r 0} \alpha_{r}
$$

with the tire cornering stiffness $c_{f 0}, c_{r 0}$, the road adhesion factor $\mu$, and the tire side slip angles

$$
\begin{aligned}
\alpha_{f} & =\delta_{f}-\left(\beta+\frac{\ell_{f}}{v} r\right) \\
\alpha_{r} & =-\left(\beta-\frac{\ell_{r}}{v} r\right)
\end{aligned}
$$

A variable of great importance in lateral vehicle dynamics is the lateral acceleration at the front axle, $a_{y f}$. It can be easily shown that,

$$
a_{y f}=v(\dot{\beta}+r)+\ell_{f} \dot{r} .
$$

For steer-by-wire control the essential characteristic of vehicle dynamics is its lateral impedance, which will be defined as the ratio between the front lateral reaction force, $F_{f}$ and the front wheel steering angle, $\delta_{f}$, $Z_{v}=F_{f} / \delta_{f}$. In this paper, for the sake of simplicity, the single-track model will be used to compute it. By combining the equations (50), (51), (52) and (53) it can be shown that

$$
Z_{v}(s)=\frac{a_{2} s^{2}+a_{1} s+a_{0}}{s\left(b_{2} s^{2}+b_{1} s+b_{0}\right)}
$$

with

$$
\begin{aligned}
& a_{2}=c_{f} \mu v^{2} m J \\
& a_{1}=\mu^{2} c_{f} c_{r} v\left(J+m \ell_{r}^{2}\right)
\end{aligned}
$$




$$
\begin{aligned}
& a_{0}=c_{f} c_{r} \ell_{r} \mu^{2} v^{2} m \\
& b_{2}=m v^{2} J \\
& b_{1}=\mu\left(\left(c_{f}+c_{r}\right) J+m\left(c_{f} \ell_{f}^{2}+c_{r} \ell_{r}^{2}\right)\right) \\
& b_{0}=\mu\left(\mu c_{r} c_{f}\left(\ell_{f}+\ell_{r}\right)^{2}-m v^{2}\left(c_{f} \ell_{f}-c_{r} \ell_{r}\right)\right) .
\end{aligned}
$$

Notice that, $Z_{v}(s, \mu, v)$ is uncertain because of the uncertainties in physical parameters $\mu$ and $v$.

\subsection{Open Actuation Loops}

This section is about setting up an open-loop model description of a steer-by-wire actuation system. As already noted, a steer-by-wire control system includes two actuation inner-loops for torque and/or position control. The dynamics of the force feedback loop can in general be described by the linear equation

$$
\dot{\delta}_{l}=y_{l}(s)\left(T_{l}-\alpha_{l}(s) \tau_{m}\right)
$$

which formally describes both, the closed-loop and open-loop force feedback. Notice that the dynamics of the actuator, sensor and controller are lumped into the transfer functions $\alpha_{l}$.

Similarly, in an admittance steer-by wire structure the road-wheel actuation loop is described by the equation

$$
\dot{x}_{r}=y_{r}(s)\left(F_{r}-\alpha_{r}(s) f_{s}\right)
$$

while in a hybrid steer-by-wire structure it is described by

$$
F_{r}=z_{r}\left(\dot{x}_{r}-\beta_{r} \dot{x}_{s}\right) .
$$

\section{Thereby}

$\dot{x}_{r}: \quad$ rack position rate

$F_{r}: \quad$ rack force (road-feedback)

$\dot{x}_{s}$ : reference rack-position rate

$f_{s}$ : reference road-wheel actuation force.

By introducing the following definitions

$$
v_{a}=\left[\begin{array}{c}
\dot{\delta}_{l} \\
\dot{x}_{r}
\end{array}\right] \quad r_{a}=\left[\begin{array}{c}
T_{l} \\
F_{r}
\end{array}\right] \quad \tau_{1}=\left[\begin{array}{c}
\tau_{m} \\
f_{s}
\end{array}\right]
$$

and

$$
v_{l}=\left[\begin{array}{c}
\dot{\delta}_{l} \\
F_{r}
\end{array}\right] \quad r_{l}=\left[\begin{array}{c}
T_{l} \\
\dot{x}_{r}
\end{array}\right] \quad \tau_{2}=\left[\begin{array}{c}
\tau_{m} \\
\dot{x}_{s}
\end{array}\right] .
$$

The dynamics of the open-loop admittance steer-bywire structure can be described by the equation

$$
v_{a}=Y_{e}(s)\left(r_{a}-A_{1}(s) \tau_{1}\right)
$$

with

$$
Y_{e}=\left[\begin{array}{cc}
Y_{l e}(s) & 0 \\
0 & Y_{r e}(s)
\end{array}\right] \quad A_{1}=\left[\begin{array}{cc}
\alpha_{l}(s) & 0 \\
0 & \alpha_{r}(s)
\end{array}\right]
$$

Similarly open-loop dynamics of the hybrid steer-bywire structure may be modelled by the equation

$$
v_{l}=H\left(r_{l}-A_{2} \tau_{2}\right)
$$

whereby

$$
H=\left[\begin{array}{cc}
y_{l} & 0 \\
0 & z_{r}
\end{array}\right] \quad A_{2}=\left[\begin{array}{cc}
\alpha_{l} & 0 \\
0 & \beta_{r}
\end{array}\right] .
$$

\subsection{Model-Matching Based Design}

Basically, four different control structures may be applied for a steer-by-wire interaction. Depending on feed-backed variables, one can discriminate between the admittance, hybrid and impedance control topology.

In an admittance system description, (46), the steering wheel angle rate, $\dot{\delta}_{l}$ and the rack position rate, $\dot{x}_{r}$ are the measured/controlled variables. The controller structure in this case is

$$
\left[\begin{array}{c}
\tau_{m} \\
f_{s}
\end{array}\right]=C_{y}(s)\left[\begin{array}{c}
\dot{\delta}_{l} \\
\dot{x}_{r}
\end{array}\right]+\left[\begin{array}{c}
0 \\
f_{A}
\end{array}\right]
$$

The primary advantage of this structure is its simple sensory requirement.

In a hybrid steer-by-wire structure the rack force, $F_{r}$, is assumed to be known instead of the rack position rate, $\dot{x}_{r}$. Its controller structure has the following form

$$
\left[\begin{array}{c}
\tau_{m} \\
\dot{x}_{s}
\end{array}\right]=C_{l}\left[\begin{array}{c}
\dot{\delta}_{l} \\
F_{r}
\end{array}\right]+\left[\begin{array}{c}
0 \\
\dot{x}_{A}
\end{array}\right] .
$$

This paper considers the controller structure in (65), whereby the same formalism can be applied to that in (66)

By closing the loop in (61) and using (65) it follows

$$
v_{a}=Y\left(r_{a}-A_{1} C_{y} v_{a}\right)
$$


Its solution $v_{a}$ is

$$
v_{a}=\left(I+Y_{e} A_{1} C_{y}\right)^{-1} Y_{e} r_{a} .
$$

The control problem may be formulated as follows: find the controller $C_{y}$ such that the closed-loop response $r_{a} \rightarrow v_{a}$ resembles the steering dynamics described by (46), i.e.

$$
\left\|\left(I+Y_{e} A_{1} C_{y}\right)^{-1} Y_{e}-Y_{d}\right\|_{\infty}=\text { minimal }
$$

whereby $Y_{d}$ corresponds to the desired admittance matrix in (46). In order to solve this problem the controller $C_{y}$ is parameterized using Youla parameterization (Doyle et al., 1992; Francis, 1987)

$$
C_{y}=(B-M Q)(A-N Q)^{-1}
$$

whereby matrices $M$ and $N$ coprime-factorize the product $Y A_{1}$

$$
Y A_{1}=N M^{-1}=\tilde{M}^{-1} \tilde{N}
$$

and

$$
\left[\begin{array}{cc}
\tilde{A} & -\tilde{B} \\
-\tilde{N} & \tilde{M}
\end{array}\right]\left[\begin{array}{ll}
M & B \\
N & A
\end{array}\right]=I .
$$

$Q$ belongs to Hardy space, $\mathcal{R} \mathcal{H}_{\infty}$, of all proper and real rational stable transfer matrices and represents the free parameter. Using the last three equations, after some algebraic operations, (69) may be transformed to the model-matching problem

$$
\left\|T_{1}-T_{2} Q T_{3}\right\|_{\infty}=\text { minimal }
$$

whereby

$$
\begin{aligned}
T_{1} & =A \tilde{M} Y_{e}-Y_{d} \\
T_{2} & =N \tilde{M} \\
T_{3} & =\tilde{M} Y_{e} .
\end{aligned}
$$

The model-matching problem may be solved using LMI tools or some other $H_{\infty}$ optimization tool. The target steer-by-wire admittance $Y_{d}$ used in this article is

$$
\begin{aligned}
& y_{11}=\frac{33.33 s^{2}+448.7 s+93690}{s^{3}+20.71 s^{2}+7655 s+45790} \\
& y_{12}=\frac{1.295 s+894.6}{s^{3}+20.71 s^{2}+7655 s+45790}
\end{aligned}
$$

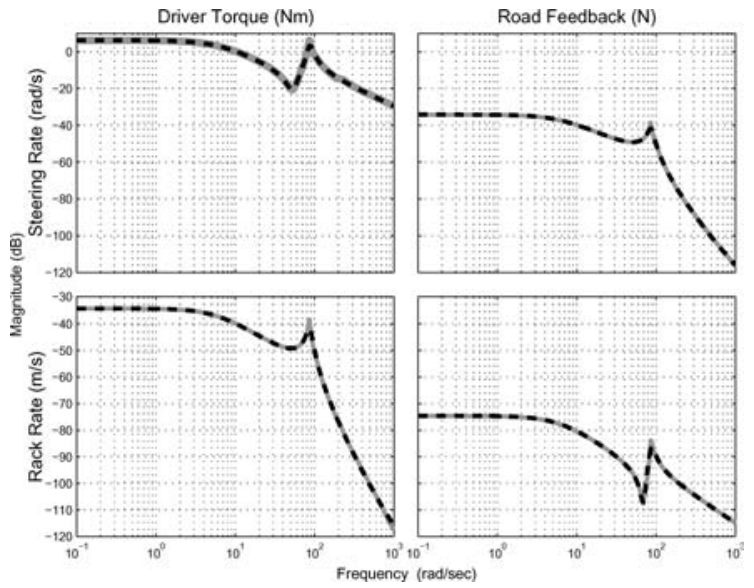

Figure 15. Comparison of desired steer-by-wire (solid line) dynamics and closed-loop dynamics (dashed line).

$$
\begin{aligned}
& y_{21}=\frac{-1.295 s-894.6}{s^{3}+20.71 s^{2}+7655 s+45790} \\
& y_{22}=\frac{-0.001789 s^{2}-0.01296 s-8.543}{s^{3}+20.71 s^{2}+7655 s+45790}
\end{aligned}
$$

which represents the linear part of a conventional steering system. Further the open-loop actuation is described by transfer functions

$$
\begin{aligned}
y_{l} & =\frac{26.67 s^{2}+568 s+218300}{s^{3}+28.1 s^{2}+16430 s+120000} \\
y_{r} & =\frac{-0.00179}{s+9.395} \\
\alpha_{l} & =\frac{-13510}{s^{2}+21.3 s+8188} \\
\alpha_{r} & =\frac{1974000}{s+1885}
\end{aligned}
$$

In Fig. 15 the frequency response of designed linear reference steer-by-wire system and closed-loop dynamics are compared.

\subsection{Steer-by-Wire AOB Design}

This section provides the design of a steer-by-wire controller using the AOB algorithm. The target model of a conventional vehicle within the framework of $\mathrm{AOB}$ 
design is given by

$$
\left[\begin{array}{c}
\dot{\delta}_{l} \\
\dot{x}_{r}
\end{array}\right]=\left[\begin{array}{llll}
y_{11} & 0 & y_{12} & 0 \\
y_{21} & 0 & y_{22} & 0
\end{array}\right]\left[\begin{array}{c}
T_{l} \\
\tau_{m} \\
F_{r} \\
f_{s}
\end{array}\right]
$$

Note that the inputs $T_{l}$ and $F_{r}$ are not directly reached by actuators. The model-matching problem in the AOB framework consists in designing a state-space controller for the open-loop system

$$
\left[\begin{array}{c}
\dot{\delta}_{l} \\
\dot{x}_{r}
\end{array}\right]=\left[\begin{array}{cccc}
y_{l} & -\alpha_{l} y_{l} & 0 & 0 \\
0 & 0 & y_{r} & -\alpha_{r} y_{r}
\end{array}\right]\left[\begin{array}{c}
T_{l} \\
\tau_{m} \\
F_{r} \\
f_{s}
\end{array}\right]
$$

to achieve (85). Writing (85) in discrete statespace form with sampling time $T_{s}=1[\mathrm{~ms}]$, the desired closed-loop poles result at $\lambda_{1,2}=0.9890 \pm$ $0.0859 i$ and $\lambda_{3}=0.9940$.

The discrete state-space of (86) can be represented by (35), where $\phi_{r}$ and $\Gamma_{r}$ have dimensions $7 \times 7$ and $7 \times 4$, respectively. To apply the AOB design described in Section 4, a state feedback matrix $L_{r}$ has to be found. In addition to the three above desired closed-loop poles, four additional poles $\left(p_{1} \ldots p_{4}\right)$ are needed due to the dimension of $\Phi_{r}$. There is no straightforward procedure to obtain the additional poles. Simulations have shown that there are many possibilities that guarantee good results, such as $p_{1}=0.89, p_{2}=0.89, p_{3}=$ -0.89 and $p_{4}=0.994$. The minus sign in one pole is important due to the transient effect of a positive zero in the target system. To compute $L_{r}, \Gamma_{r}$ has to be changed (only for this computation) to reflect the lack of actuation on the inputs $T_{l}$ and $F_{r}$. The first and third columns of $\Gamma_{r}$ should be set to zero. This guarantees that if there is an $L_{r}$, it will not generate a direct feedback referred to non-actuated inputs. The DC gain of the steer-by-wire system is compensated by proper pre-amplification of the reference inputs. For the target system $\left({ }^{\prime} t^{\prime}\right.$ stands for target system)

$$
\mathrm{DC}_{t}=C_{t}\left[I-\left(\phi_{t}\right)\right]^{-1} \Gamma_{t}
$$

and for the steer-by-wire system

$$
\mathrm{DC}_{S b W}=C_{S b W}\left[I-\left(\phi_{S b W}-\Gamma_{S b W} L_{S b w}\right)\right]^{-1} \Gamma_{S b W} .
$$

Therefore

$$
L_{c}=\left(\mathrm{DC}_{S b W}\right)^{-1} \quad \mathrm{DC}_{t}
$$

i.e.

$$
L_{c}=\left[\begin{array}{cc}
0.4785 & -0.0046 \\
67.0076 & -0.6399
\end{array}\right] \text {. }
$$

The reference input is then

$$
r_{k}=L_{c}\left[\begin{array}{l}
T_{l} \\
F_{r}
\end{array}\right]
$$

$A O B$ estimation strategies for steer-by-wire. Model reference adaptive control appears if $Q_{x_{r, k}}$ is much smaller than $Q_{p_{k}}$. In this case, the estimation for the system state follows the reference model. Everything that does not fit in the $x_{r, k}$ model goes to $p_{k}$. Knowing the structure of $Q_{k}$, the relation between $R_{k}$ and $Q_{k}$ makes the estimates more ( $R_{k}$ low) or less ( $R_{k}$ high) sensitive to measures. The stochastic parameters of $Q_{k}$ and $R_{k}$ are a powerful tool in the control design, creating enough space to explore complex estimation strategies for highly unstructured tasks. For the steer-by-wire setup

$$
\begin{aligned}
Q_{x_{r, k}} & =10^{-12} I_{7 \times 7} \\
Q_{p_{k}} & =\left[\begin{array}{cccc}
0 & 0 & 0 & 0 \\
0 & 10^{-1} & 0 & 0 \\
0 & 0 & 0 & 0 \\
0 & 0 & 0 & 10^{3}
\end{array}\right]
\end{aligned}
$$

$R_{k}=4 I_{2 \times 2}$ and $P_{0}=Q_{0}$. Since there are four inputs, $p_{k}$ has four states $p_{k}=\left[\begin{array}{llll}p_{1, k} & p_{2, k} & p_{3, k} & p_{4, k}\end{array}\right]^{T} \cdot p_{1, k}$ and $p_{3, k}$ cannot enter in the system (there is no actuation entry). Hence, the first and third lines of $Q_{p_{k}}$ are zero. This design inactivates the estimation of $p_{1, k}$ and $p_{3, k}$ $\left(p_{1, k}=0\right.$ and $\left.p_{3, k}=0\right)$. In the steer-by-wire design, the uncertainty of $p_{4, k}$ is higher than $p_{2, k}$, enabling $p_{4, k}$ to have faster dynamics.

\subsection{Simulation Results}

This section shows simulation results of a steer-bywire system with the linear model-matching controller, whereby its dynamics is coupled to the standard singletrack vehicle model for vehicle dynamics simulation. The responses of the closed-loop steer-by-wire system and target steering systems with respect to two input 

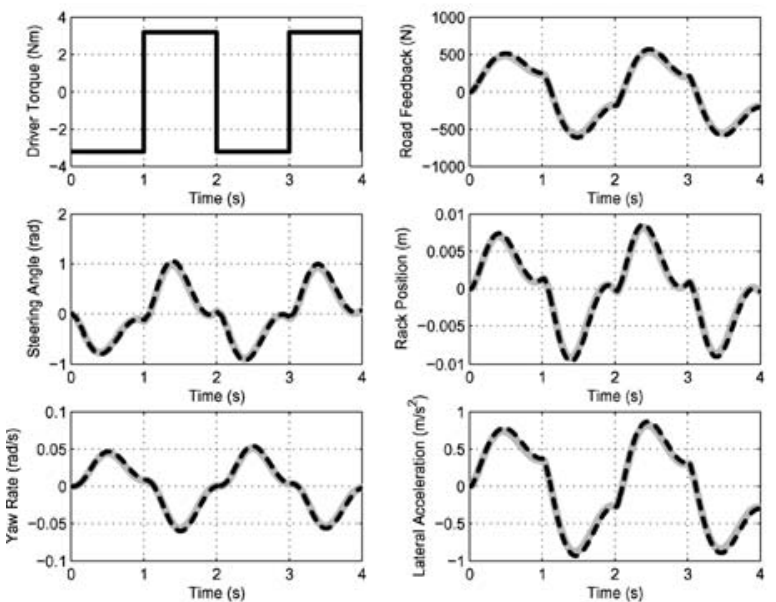

(a) $v=80 \mathrm{~km} / \mathrm{h}, \mu=0.8$
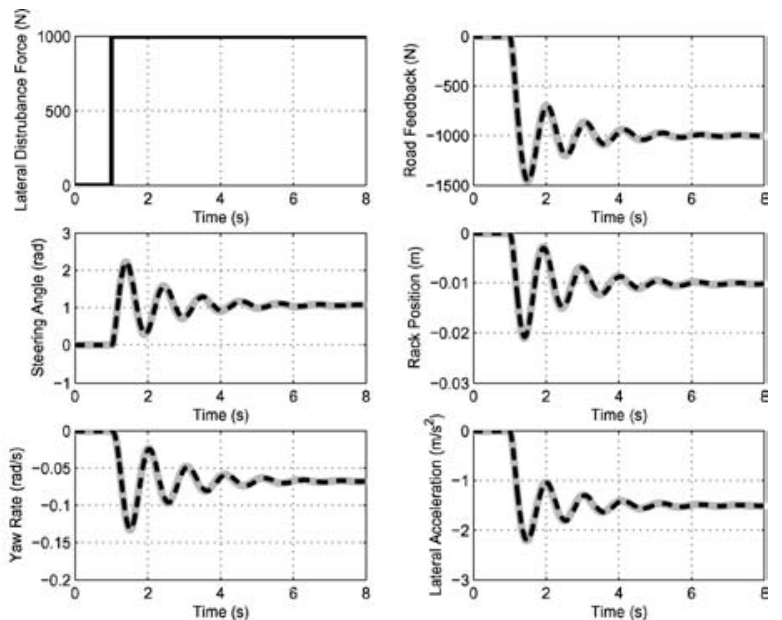

(b) $v=80 \mathrm{~km} / \mathrm{h}, \mu=0.8$

Figure 16. Responses of linear steer-by-wire vehicle (dashed line) and conventional vehicle (solid line) to rectangular periodic driver input torque (left) and disturbance force on steering link (right).
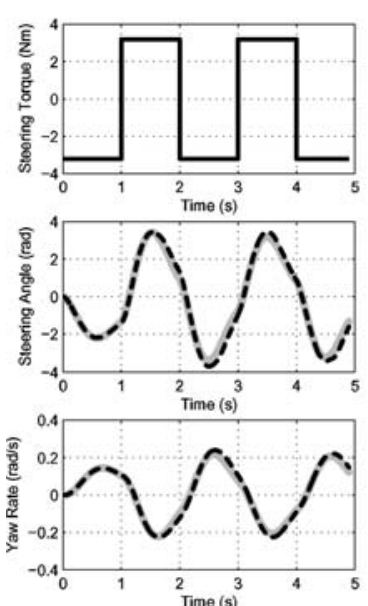

(a) $v=80 \mathrm{~km} / \mathrm{h}, \mu=0.8$
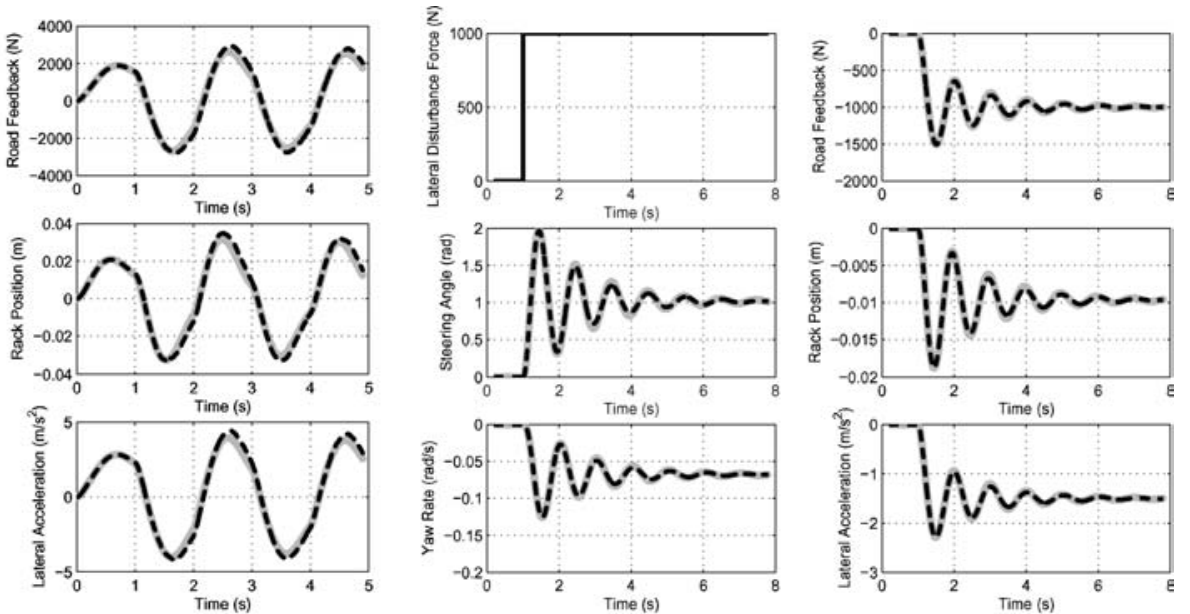

(b) $v=80 \mathrm{~km} / \mathrm{h}, \mu=0.8$

Figure 17. Responses of non-linear steer-by-wire vehicle (dashed line) and EPAS vehicle (solid line) to rectangular periodic driver input torque (left) and disturbance force on steering link.

scenarios are compared: (a) the driver torque on the steering wheel is a periodic rectangular signal of frequency $0.5 \mathrm{~Hz}$ and amplitude $3.2 \mathrm{Nm}$, and (b) a stepwise disturbance force of amplitude $1 \mathrm{kN}$ is applied on the steering link. Comparisons for both: linear (no force assistance, Fig 16) and non-linear (with force assistance, Fig 17) systems are done in terms of responses of the steering wheel angle $\delta_{l}$, steering rack position $x_{r}$, road-feedback, $F_{r}$, and vehicle dynamics variables: yaw rate, $r$, and lateral front-wheel acceleration, $a_{y f}$.

\section{Robustness Analysis}

\subsection{Passivity Approach}

Passivity provides the basic framework of state of the art methods used for design and analysis of teleoperation systems. Thus, it is important to investigate its usability for steer-by-wire.

7.1.1. Basic Definitions. This subsection recalls briefly some very basic definitions on passivity, which 
will be used on later discussions, (Sepulchre et al., 1997; Khalil, 1996; Slotine and Lee, 1991). Consider a system $H$ with an input vector $u$ and output vector $y$ of the same dimension $m$, whereby $u: \mathbb{R}^{+} \mapsto \mathbb{R}^{m}$ is bounded. Assume further that for the system $H$ the supply rate $w: \mathbb{R}^{m} \times \mathbb{R}^{m} \mapsto \mathbb{R}$ may be defined such that $\int_{t_{o}}^{t_{1}}|w(u(t), y(t))| \mathrm{d} t<\infty, \forall t_{o} \leq t_{1}$. The system $H$ is said to be dissipative with the supply rate $w(u, y)$ if there exists a function of states $x(t), S(x) \geq 0$, $S(0)=0$, which is called storage function such that

$$
S(x(T))-S(x(0)) \leq \int_{0}^{T} w(u(t), y(t)) \mathrm{d} t
$$

$\forall T \geq 0$. Further, $H$ is said to be passive if it is dissipative with supply rate $w(u, y)=u^{T} y$.

Assume a system divided in a set of subsystems, such that its storage function is the sum of the storage functions of the subsystems. The whole system may still be passive, even if some its subsystem is not passive. In other words, one can assign to a system the notions shortage and excess of passivity. The system $H$ is said to be output feedback passive if it is dissipative with respect to $w(u, y)=u^{T} y-\rho y^{T} y$ for some $\rho \in \mathbb{R}$. Analogously, $H$ is said to be input feedback passive if it is dissipative with respect to $w(u, y)=u^{T} y-\eta u^{T} u$ for some $\eta \in \mathbb{R}$. Accordingly, positive sign of $\rho$ and $\eta$ means that the system has an excess of passivity, and conversely, negative sign of $\rho$ and $\eta$ means that the system has a shortage of passivity.

7.1.2. Robust Stability. Figure 18 represents a master-slave observation of a steer-by-wire system. $Z_{d}$ represents the impedance of the arm of the driver (master) and $Z_{v}$ the lateral vehicle impedance (environment), i.e. the friction force of the tyre-road friction due to the rack rate $\dot{x}_{r}$. The two impedances interact via the steer-by-wire actuation and control algorithm. Notice that the forces generated at the driver arm muscles and lateral vehicle disturbances are ignored.

A well-known passivity-based theorem on the robust stability of such a master-slave structure is: given the passivity of the master and environment impedance, the whole master-slave system is robust stable iff the block Actuation + Control is passive. Notice that no other assumptions regarding the master and environment dynamics are assumed besides being passive.

7.1.3. Passivity Bounds in Parameter Space. This work aims to analyze the stability and/or passivity re-

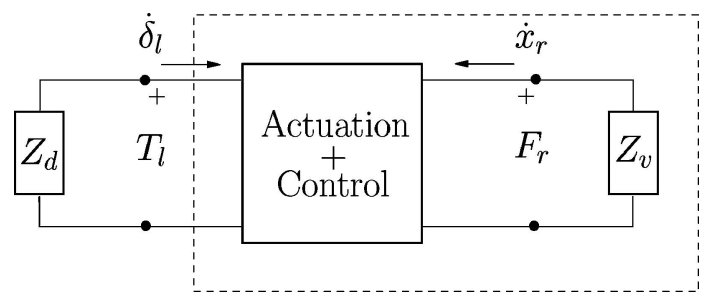

Figure 18. Master-slave representation of steer-by-wire interaction.

gions in the space of physical parameters of a steer-bywire system, such as driver arm stiffness, $C_{d}$, vehicle speed, $v$, and tyre-road adhesion coefficient $\mu$. To this end, the approach of mapping of specifications in parameter space will be used. This section provides a brief introduction to the method used therefore.

Assume $Y(s, \boldsymbol{q})$ is a given transfer function matrix, which depends on some parameters $\boldsymbol{q}$, which may include uncertain physical parameters or/and controller parameters. Transfer function matrix $Y(s, \boldsymbol{q})$ is passive at some $\boldsymbol{q}$ iff the hermitian matrix, (Slotine and Lee, 1991),

$$
H(j \omega, \boldsymbol{q})=Y(j \omega, \boldsymbol{q})+Y^{T}(-j \omega, \boldsymbol{q})
$$

is positive-definite for each $\omega$ at $\boldsymbol{q}$.

The following is standard in linear algebra. A hermitian matrix $H(j \omega)=\left(h_{i j}\right)$ is positive definite iff

$$
\Delta_{k}^{(H)}(j \omega)>0, \quad \forall \omega>0, k=1,2, \ldots, m
$$

with

$$
\Delta_{k}^{(H)}(j \omega)=\left|\begin{array}{ccc}
h_{11}(j \omega) & \cdots & h_{1 i}(j \omega) \\
\vdots & & \vdots \\
h_{i 1}(j \omega) & \cdots & h_{i i}(j \omega)
\end{array}\right|
$$

Now define

$$
\boldsymbol{Q}_{\pi}^{(k)}=\left\{\boldsymbol{q}: e_{k}(\omega, \boldsymbol{q}) \doteq \Delta_{k}^{(H)}(\omega, \boldsymbol{q}) \geq 0\right\} .
$$

Then the parameter region which drives $Y(j \omega, \boldsymbol{q})$ passive is the intersection

$$
\boldsymbol{Q}_{\pi}=\bigcap_{k} \boldsymbol{Q}_{\pi}^{(k)}
$$

Thus, finding the passivity bounds of the whole matrix is reduced to solving a system of $m$-inequalities. It can 
be shown that the bounds of the set $\boldsymbol{Q}_{\pi}^{(k)}$ are defined by the solution of the two following parametric nonlinear equations

$$
e_{k}(\omega, \boldsymbol{q})=0, \quad \frac{\partial e_{k}}{\partial \omega}(\omega, \boldsymbol{q})=0
$$

Notice that, this methodology is convenient if the vector $\boldsymbol{q}$ includes few parameters.

7.1.4. Example: Lateral Vehicle Impedance. Figure 19 shows the passivity bounds of the lateral vehicle impedance in the parameter plane $(\mu, v)$.

Note that, unlike the usual tele-operation applications, a steer-by-wire system comprehends a nonpassive environment, which complicates to some extent the robust stability considerations, since the conditions of the cited theorem in Section 1.2 are not fulfilled. In fact, physically this is not a strange condition, since for velocities $v \neq 0$, when steering, an amount of longitudinal kinetic energy is sent to the lateral dynamics. (Notice, for $v=0$ the vehicle is passive, which is intuitive.) For a given friction coefficient, $\mu$, it can be shown that the critical vehicle speed when passivity properties switch is

$$
v_{c r}=\sqrt{\frac{\mu c_{r}\left(\ell_{f}+\ell_{r}\right)\left(J+\ell_{r}^{2} m\right)}{m\left(J+\ell_{f} \ell_{r} m\right)}} .
$$

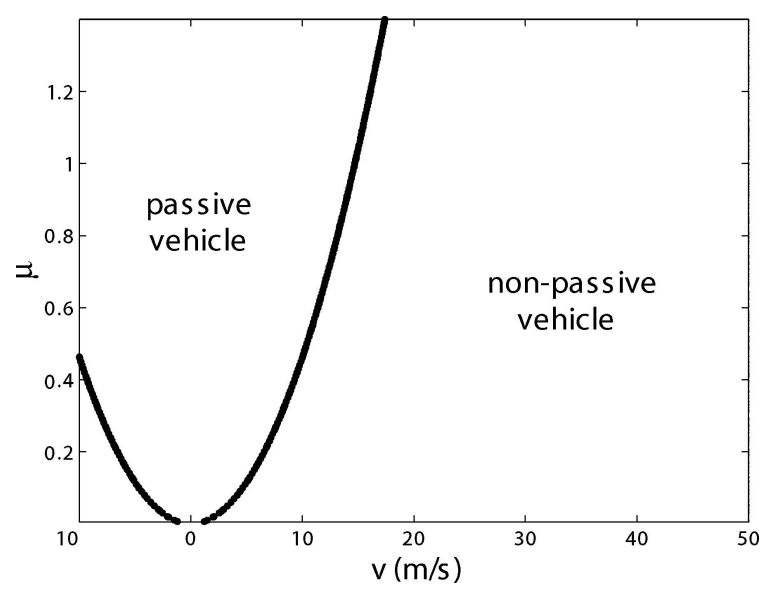

Figure 19. Passivity bounds of the open-loop lateral vehicle impedance, $Z_{v}$.

\subsection{Robust Passivity}

7.2.1. Linear Steer-by-Wire System. Assume that the steer-by-wire controller is passive. The modelmatching controller designed in this paper, indeed shows output passivity excess. Hence, it is interesting to investigate the impact of such an excess into the passivity property of the feedback loop designated by the dashed line in Fig. 18. Because of the passivity excess of the controller, it is to be expected that the passivity region of the feedback loop will increase compared to that shown in Fig. 19. In Fig. 20 passivity bounds of the feedback loop are computed. Notice that the passivity excess of the controller can compensate for the activity of the vehicle in the region of small $\mu$. Nevertheless, passivity in the whole operating domain of a vehicle is not provided. Hence, for robust passivity a new controller has to be designed (probably) at the price of performance, i.e. of model-matching.

7.2.2. Non-Linear Steer-by-Wire System. Consider the non-linear steer-by-wire vehicle with force assistance, Fig. 21. ${ }^{4}$ Such a structure provides an additional power source, so it is intuitively clear that passivity shortage of the feedback structure will be further increased. According to the previous discussion, the linear steer-by-wire system has already output passivity shortage, i.e.

$$
\int_{0}^{T}\left(u^{T} y+\rho y^{T} y\right) \mathrm{d} t \geq S(x(T))-S(x(0))
$$

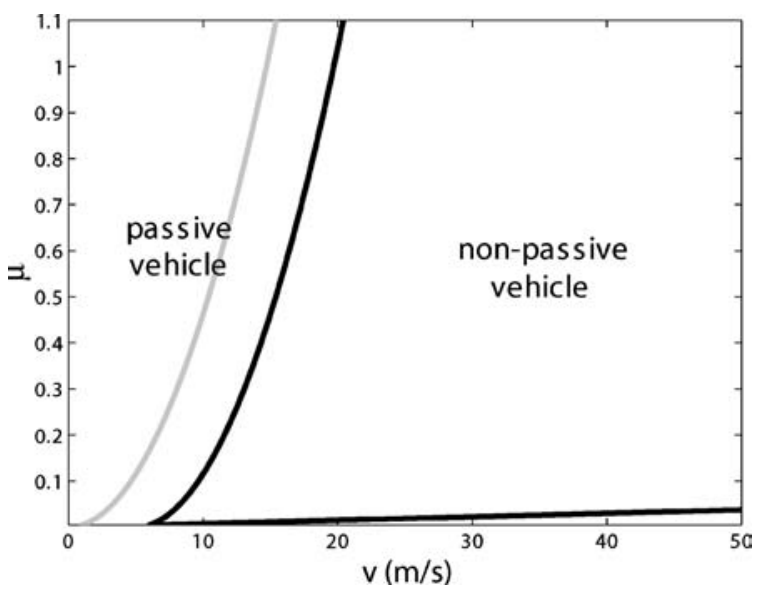

Figure 20. Passivity bounds of the coupling between steer-by-wire steering system and lateral vehicle dynamics. 


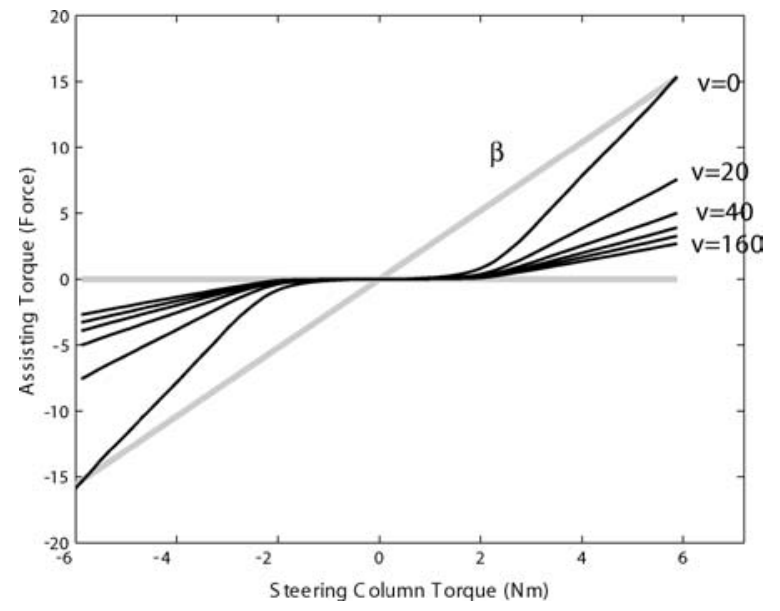

Figure 21. Boost curve and its dependence on vehicle speed.

whereby $\rho>0$. Since the dynamics of the boost-curve, Fig. 21, is void, the storage function of the feedback loop is equal to that of the open-loop, $S(x)$. Substitution of

$$
u=r+\psi(y)
$$

in (98), whereby, $\beta|y|>|\psi(y)|, \forall y$, Fig. 21, yields the condition of passivity of the closed-loop

$$
\begin{aligned}
& \int_{0}^{T}\left(r^{T} y-\left(-\psi(y)^{T} y-\rho y^{T} y\right)\right) \mathrm{d} t \\
& \quad \geq S(x(T))-S(x(0))
\end{aligned}
$$

which further requires an impossible condition

$$
-\beta-\rho>0
$$

Thus, it is to be concluded that passivity tools fail to prove robust stability of steer-by-wire systems.

\subsection{Robust Stability}

This section presents an alternative approach for analysis of robust stability of steer-by-wire systems with respect to parameter uncertainties. Again the aim is to find the stability regions in the space of uncertain parameters.

7.3.1. Linear Steer-by-Wire System. Consider the linear system shown in Fig. 22 with the feedback loop containing the uncertainties of a steer-by-wire system.

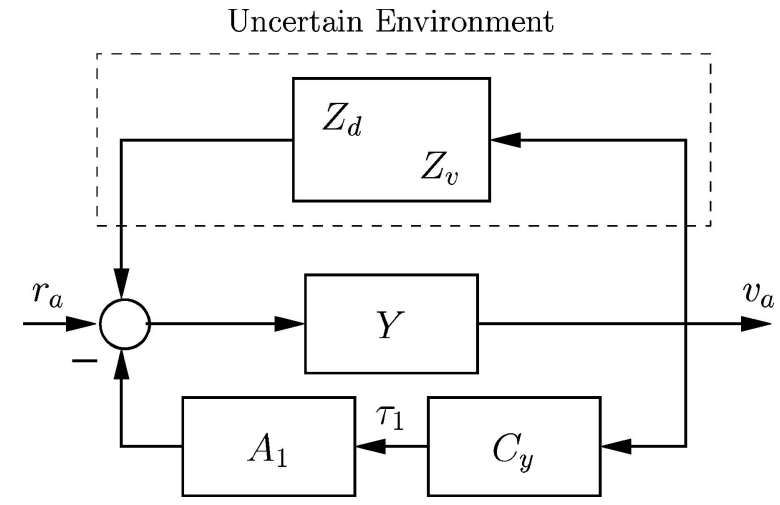

Figure 22. Linear steer-by-wire system in feedback with the uncertain environment.

The main uncertain parameters are vehicle speed $v$ friction coefficient $\mu\left(Z_{v}=Z_{v}(s, v, \mu)\right)$, and driver stiffness $C_{d}\left(Z_{d}=Z_{d}\left(C_{d}\right)\right)$.

A straightforward method is to compute the characteristic polynomial of the system, which describes the eigenvalues of the steer-by-wire system in dependence of uncertain parameters. By doing this, and separating the characteristic equation into its real and imaginary part, once again is met the system of two parametric nonlinear equations

$$
h\left(\omega, v, \mu, C_{d}\right)=0, \quad g\left(\omega, v, \mu, C_{d}\right)=0
$$

whereby $0<\omega<\infty$ stands for the Hurwitz frequency and represents the gridding parameter of the equations. Figure 23 shows its solution. An interesting fact is that for a given vehicle dynamics operating point, i.e. $v=$ const and $\mu=$ const, the stability radius increases with increasing driver stiffness $C_{d}$. The curves in Fig. 23 represent the stability bounds for a linear model, which neglects the non-linearities (e.g. gearing friction) in the system. However they provide insight on the stability robustness with respect to parameters uncertainties.

7.3.2. Nonlinear Steer-by-Wire System. Now consider Fig. 24 with the boost-curve feedback. In nonlinear control the global asymptotic stability of this structure is denoted as absolute stability of $H$, with respect to the sector $(0, \beta)$ static nonlinearity. For its investigation in parameter space, in this paper the circle criterion, (Sepulchre et al., 1997; Khalil, 1996), will be used, since it fits well within the analysis framework developed in Section 7.1 of mapping of 


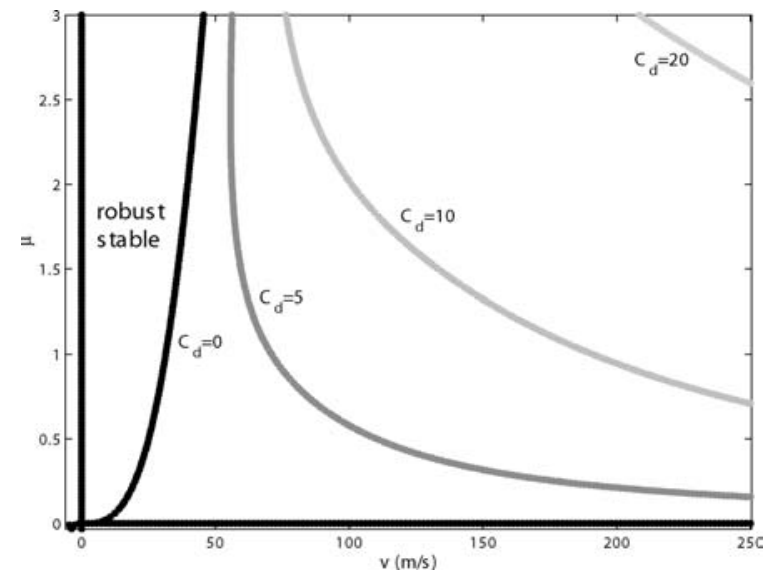

Figure 23. Robust stability of linear steer-by-wire system.

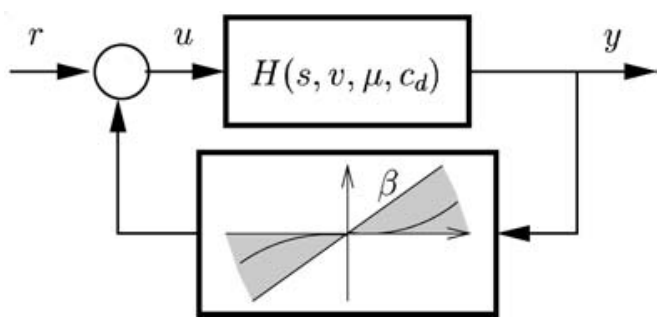

Figure 24. Absolute stability of non-linear steer-by-wire system with boost-curve feedback.

positive realness bounds in parameter space. According to the circle criterion, the feedback loop of a linear system $H(s)$ with any static nonlinearity of the sector $(0, \beta)$ is global asymptotic stable, if the transfer function

$$
\tilde{H}(s)=H(s)-\frac{1}{\beta}
$$

is positive real $(\beta>0)$. In the case of steer-by-wire system, the linear part is further uncertain, thus extending the requirement for robust absolute stability with respect to uncertainties $v, \mu$ and $C_{d}$. Based on the discussions in Section 7.1, the mapping equations, which correspond to circle criterion are directly derived to be

$$
e\left(\omega, v, \mu, C_{d}\right)=1 / \beta, \quad \frac{\partial e}{\partial \omega}\left(\omega, v, \mu, C_{d}\right)=0 .
$$

Thus, by tuning $\beta$, bounds of robust absolute stability in the space of parameters $\left(v, \mu, C_{d}\right)$ are gained. Notice that this approach provides good insights also for the practical design of the boost-curve, since the required stability radius for a given $\beta$ may be clearly read in charts similar to that shown in Fig. 23.

\section{Conclusions}

At the actuation level robust force control algorithms for steer-by-wire vehicles are developed using inverse disturbance observer (IDOB) control scheme and active observer algorithms (AOB). Simulation and experimental results validate both proposed structures.

Methods for design and analysis of steer-by-wire systems at the operational level are further presented. Model-matching approach is shown to be an appropriate method, once the desired steering dynamics is known. Therefore the controllers have been developed using $H_{\infty}$ and AOB formalisms. The design methods are illustrated on the admittance steer-by-wire structure, but they can be applied equally well for the hybrid structure. Further, methods for the analysis of robust stability of a given steer-by-wire system with respect to the uncertain physical parameters are introduced. They apply to both, linear and nonlinear steer-by-wire systems with static nonlinearities.

\section{Notes}

1. The general AOB algorithm uses $N$ extra states to describe $p_{k}$ (Cortesão, 2003).

2. For the sake of simplicity we do not consider here the effects resulting form uncertainties in the driver arms inertia $J_{d}$.

3. The parameter values of the linearized single track model assumed in this paper are $l_{f}=1.25 \mathrm{~m}, l_{r}=1.32 \mathrm{~m}, m=1296 \mathrm{~kg}$, $J=1750 \mathrm{~kg} \mathrm{~m}^{2}, c_{f 0}=9244 \mathrm{~N} / \mathrm{rad}$ and $c_{r 0}=105750 \mathrm{~N} / \mathrm{rad}$.

4. In the figure the torque assistance is plotted. This signal is transformed to a linear force assistance through the rack-and-pinion gearing transmission coefficient. Both of the terms, force and torque transmissions are used in this paper.

\section{References}

Bajçinca, N. and Bünte, T. 2005. A novel control sturcture for inversion and tracking tasks. XVI-th IFAC World Congress, Prague.

Bajçinca, N., Cortesão, R., Hauschild, M., Bals, J., and Hirzinger, G. 2003a. Haptic control for steer-by-wire systems. Int. Conf. on Intelligent Robots and Systems (IROS), USA, 20042009.

Bajçinca, N., Hauschild, M., and Cortesão, R. 2003b. Robust torque control for steer-by-wire vehicles. Int. Conf. on Advanced Robotics (ICAR), Portugal, pp. 1480-1486.

Cortesão, R. 2003. Kalman techniques for intelligent control systems: theory and robotic experiments. PhD thesis, University of Coimbra. 
Cortesão, R. and Bajçinca, N. 2004. Model-matching control for steer-by-wire vehicles with under-actuated structure. sub. in Int. Conf. on Intelligent Robots and Systems (IROS), Japan.

Doyle, J.C., Francis, B.A., and Tannenbaum, A.R. 1992. Feedback Control Theory. MacMillan Publishing Company, 113 Sylvan Avenue, Englewood Cliffs, NJ.

Francis, B.A. 1987. A Course on $H_{\infty}$ Control. Springer Verlag, lecture notes in control and information sciences edition.

Hogan, N. 1989. Controlling impedance at the man/machine interface. In Proc. of the IEEE Int. Conference on Robotics and Automation.

Hogan, N. 1990. Mechanical impedance of single- and multiarticular systems. Multiple Muscle Systems, Biomechanics and Movement Organization.

Khalil, H. 1996. Nonlinear Systems. Prentice Hall: Upper Saddle River, NJ.

Sepulchre, R., Jankovic, M., and Kokotovic, P. 1997. Constructive Nonlinear Control. Springer-Verlag Series on Communications and Control Engineering (CCES).

Slotine, J.J. and Lee, W. 1991. Applied Nonlinear Control. Prentice Hall International, Inc.

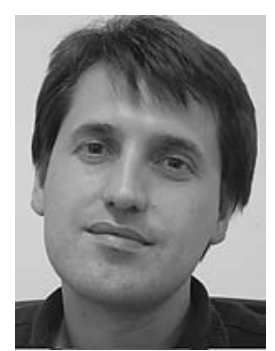

Naim Bajçinca has been working as a researcher at German Aerospace Center (DLR) in Oberpfaffenhofen since 1998. He has graduated studies on Physics (1994) and Electrical Engineering (1995) at the University of Prishtina. His main interests include methods of robust and nonlinear control, model-reference control, uncertain time-delay systems with applications on haptics, active vehicle steering and master-slave systems.

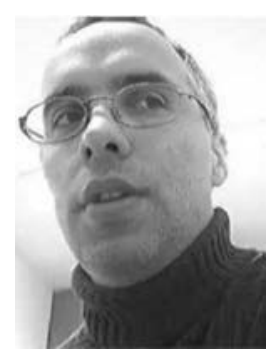

Rui Cortesão received the B.Sc. in Electrical Engineering., M.Sc. in Systems and Automation and Ph.D. in Control and Instrumentation from the University of Coimbra in 1994, 1997, and 2003, respectively. He has been visiting researcher at DLR for more than two years (1998-2003), working on compliant motion control, data fusion and steer-by-wire. In 2002 he was visiting researcher at Stanford University, working on haptic manipulation. He is Assistant Professor at the Electrical Engineering Department of the University of Coimbra since 2003 and researcher of the Institute for Systems and Robotics (ISR-Coimbra) since 1994. His research interest include data fusion, control, fuzzy systems, neural networks and robotics.

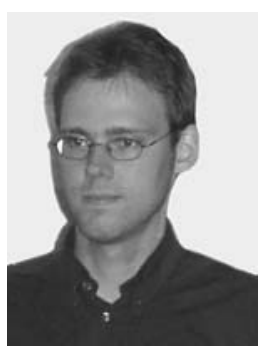

Markus Hauschild received his Diploma in Mechatronics from $\mathrm{Mu}$ nich University of Applied Sciences in 2002. He joined the DLR Institute of Robotics and Mechatronics in 2001 working on control strategies for Harmonic Drive gears. His research interests include human-machine-interfaces, x-by-wire, and modeling of biomedical systems. In 2003 he was visiting researcher at National Yunlin University of Science and Technology, Taiwan, developing iterative learning control for compensation of periodic disturbances. In the European ENACTIVE network of excellence he is the DLR coordinator for the "actuators and sensors for haptic interfaces" activities. Presently he is a PhD student at University of Southern California in the Department of Biomedical Engineering. 